\title{
Plate-Nematic Phase in Three Dimensions
}

\author{
Margherita Disertori ${ }^{1}$, Alessandro Giuliani ${ }^{2}$, Ian Jauslin ${ }^{3}$ \\ ${ }^{1}$ Institute for Applied Mathematics \& Hausdorff Center for Mathematics, University of Bonn, Bonn, Germany. \\ E-mail: disertori@iam.uni-bonn.de \\ 2 Dipartimento di Matematica e Fisica, Università degli Studi Roma Tre, Rome, Italy. \\ E-mail: giuliani@mat.uniroma3.it \\ 3 Department of Physics, Princeton University, Princeton, USA. \\ E-mail: ijauslin@ princeton.edu
}

Received: 19 May 2018 / Accepted: 10 May 2019

Published online: 5 December 2019 - (C) The Author(s) 2019

\begin{abstract}
We consider a system of anisotropic plates in the three-dimensional continuum, interacting via purely hard core interactions. We assume that the particles have a finite number of allowed orientations. In a suitable range of densities, we prove the existence of a uni-axial nematic phase, characterized by long range orientational order (the minor axes are aligned parallel to each other, while the major axes are not) and no translational order. The proof is based on a coarse graining procedure, which allows us to map the plate model into a contour model, and in a rigorous control of the resulting contour theory, via Pirogov-Sinai methods.
\end{abstract}

\section{Introduction}

The mathematical theory of liquid crystalline (LC) phases, even just of their equilibrium properties, is still in a primitive stage: most of the predictions on the phase diagram of systems of anisotropic molecules are based on density functional, or mean field theories. The approximations underlying the derivation of the corresponding effective free energy functionals are typically uncontrolled: there is no systematic way of improving the precision, and no rigorous theorem quantifying the error. Ideally, as in any equilibrium statistical mechanics problem, one would like to start from a microscopic model of interacting particles, described in terms of (say) a grand-canonical partition function at inverse temperature $\beta$ and activity $z$, and derive bounds on the large distance decay of correlations, both for the orientational and the translational degrees of freedom of the particles, for different choices of $(\beta, z)$. Given an inter-particle interaction, one would like to exhibit values of $(\beta, z)$ at which the correlation functions of the system display broken orientational order and unbroken (or partially broken) translational order. Depending on the specific nature of the broken orientational order, and/or of the unbroken/partially broken translational order, one names such a phase 'uni-axial nematic', or 'bi-axial nematic', or 'smectic', or 'chiral', etc. Essentially none of these phases has ever been mathematically proved to arise in any model of interacting particles in the 

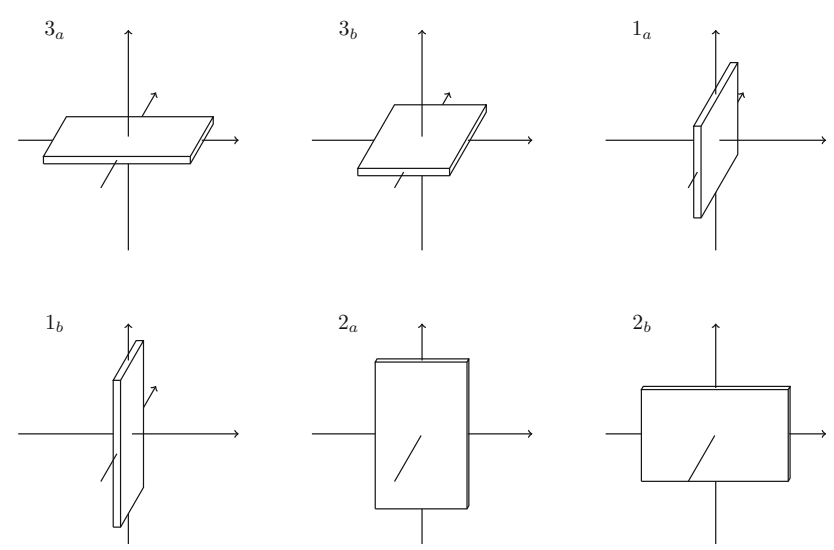

Fig. 1. The plates and their six allowed orientations

three-dimensional continuum. The purpose of this paper is to report some progress in this direction. Part of our motivation comes from the renewed interest of the condensed matter community on the nature of bi-axial nematic phases, which was stimulated by the experimental observation of a bi-axial phase in systems of elongated, boomerangshaped, particles $[1,15,16]$.

Let us specify more precisely the context we consider. As is well known, the microscopic interactions responsible for the onset of liquid crystalline phases have electrostatic origin. Electrostatic interactions among the microscopic constituents of a liquid crystal are typically strong and repulsive at short distances, and weak and attractive at larger distances (London, or Van der Waals, forces). Depending on the specific system under consideration, either the short range repulsion, or the long range attraction, plays a predominant role on the onset of the LC phase. It is customary to focus the attention on just one of the two effects, in order to understand which of those is responsible for which LC transitions, if any. Of course, if one is after quantitative results, it is important to consider both effects. In this paper, for simplicity, we focus on the effect of repulsive forces, which we model as pure hard-core interactions. As a consequence, in the model we consider, the temperature plays no role, and the only relevant parameter is the density. We also restrict our attention to the case in which the particles have a finite number of allowed orientations, which is a popular, although drastic, simplification. It is of great importance to drop this assumption and understand the phenomenon of continuous symmetry breaking in LC, as well as in other, phases of matter. We hope to report results in this direction in the future, but this goes beyond the purpose of this paper.

The model Let us now define our model more precisely: we consider a system of hard parallelepipeds of size $1 \times k^{\alpha} \times k$ for some $\alpha \in[0,1]$, which we call boards. If $\alpha<1 / 2$, a board will be called a rod and, if $\alpha>1 / 2$, a plate. The position of each board is given by the position of its center $x \in \mathbb{R}^{3}$, and its orientation, which is characterized by a pair of indices $(i, j) \in\{1,2,3\} \times\{a, b\}=: \mathcal{O}$ (see Fig. 1).

We will use the following notation: $(i, j) \equiv i_{j}$, and a board oriented along $i_{j}$ will also be said to be "in the direction $i_{j}$ ". Boards oriented in the direction $i_{a}$ or $i_{b}$ will be collectively said to be "of type $i$ ". The boards interact via a hard core interaction. We shall denote the density of board centers by $\rho$. As the density $\rho$ and the anisotropy exponent $\alpha$ are varied, the system is expected to display a variety of different phases, 


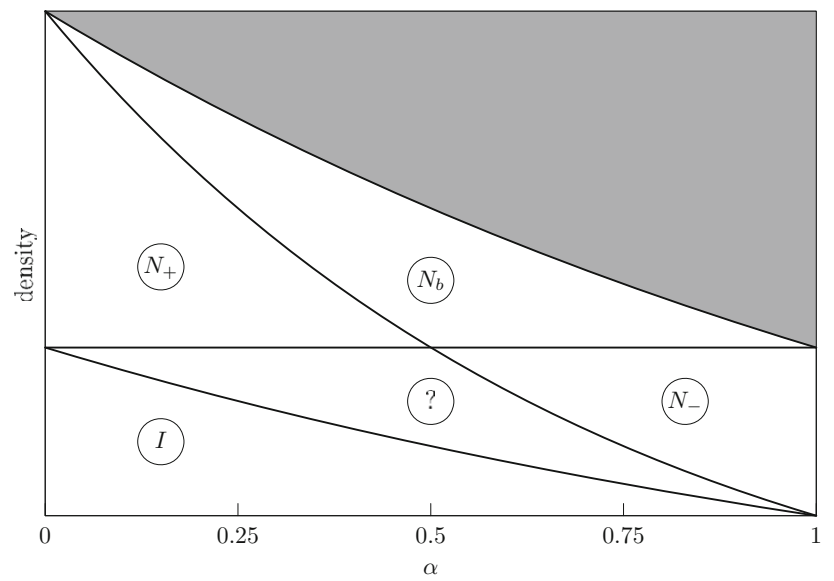

Fig. 2. Schematic phase diagram for the hard plate model. The phase labeled by $I$ is the isotropic (no orientational order), $N_{-}$is the plate-like nematic (order in the minor axis), $N_{+}$the rod-like nematic (order in the major axis), and $N_{b}$ the biaxial nematic (order in both axes). In the 'question mark' region we have no specific prediction about the nature of the phase. The region which is grayed out corresponds to densities that are too high for plates to coexist without overlapping

ranging from an isotropic liquid one, to uni-axial and bi-axial nematic, as summarized in Fig. 2.

In this paper, we focus on the case of plates, $\alpha>1 / 2$. For technical reasons, we will restrict to the sub-case $\alpha>3 / 4$; the significance of the exponent $3 / 4$ will become clear in the course of the proof. Our main result is a rigorous proof of the existence of a uni-axial nematic phase, for $k$ large, $\frac{3}{4}<\alpha \leq 1$, and the density in a suitable, $(k, \alpha)$ dependent, regime, see below for details. In principle, it should be possible to extend our analysis to smaller values of $\alpha$, most notably to the case of rods. It should also be possible to extend it to the case of larger values of the densities, thus substantiating the conjectured existence of a bi-axial nematic phase in our model. In both cases, the coarse graining procedure that we employ in the proof is insufficient for a rigorous control of the pressure and correlation functions. We hope to report progresses on the phase diagram of the system for more general values of $\rho$ and $\alpha$ in a future publication.

Before specifying our main results more precisely, let us first give a heuristic idea of why a sequence of transitions from isotropic to nematic phases is expected in our model, as the density is increased from zero to its maximum, that is $\rho_{\max }=k^{-1-\alpha}$. We focus on the case of very anisotropic plates, $\frac{1}{2}<\alpha<1$ (the 'very' stands for the condition that $\alpha<1$ ). A similar heuristic discussion can be repeated for rods, and is left to the reader.

Given a plate $(x, o) \in \mathbb{R}^{3} \times \mathcal{O}$, we define the excluded set on plates of orientation $o^{\prime}$, as the set of points $y \in \mathbb{R}^{3}$ such that the plate $\left(y, o^{\prime}\right)$ intersects the plate $(x, o)$. The excluded volume is the volume of the excluded set; it depends on the pair $\left(o, o^{\prime}\right)$. If, for instance, $o=3_{a}$, then the excluded volume on plates of different orientations are the following:

- the excluded volume produced by $3_{a}$ on $1_{a}$ is of the order $k^{2+\alpha}$,

- the excluded volume produced by $3_{a}$ on $1_{b}$ is of the order $k^{2+\alpha}$,

- the excluded volume produced by $3_{a}$ on $2_{a}$ is of the order $k^{2+\alpha}$,

- the excluded volume produced by $3_{a}$ on $2_{b}$ is of the order $k^{1+2 \alpha}$, 
- the excluded volume produced by $3_{a}$ on $3_{b}$ is of the order $k^{2}$,

- the excluded volume produced by $3_{a}$ on $3_{a}$ is of the order $k^{1+\alpha}$,

and similarly for the other choices of $\left(o, o^{\prime}\right)$. Note that for $k$ large and $\frac{1}{2}<\alpha<1$, these excluded volumes are well separated in scales, and ordered as follows: $k^{1+\alpha} \ll$ $k^{2} \ll k^{1+2 \alpha} \ll k^{2+\alpha}$. This separation of scales, together with the assumption that the distribution of the particle centers in space is approximately homogeneous, plays a prominent role in the heuristic explanation of the expected sequence of transitions. The sequence of expected transitions can be read from Fig. 2 above, and is summarized for the reader's convenience here:

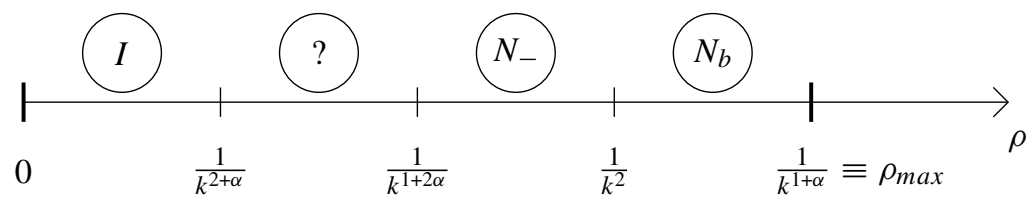

The letters $I, N_{-}$and $N_{b}$ stand for: isotropic phase, uni-axial nematic phase (the indicates that the minor axes are aligned), and bi-axial nematic phase, respectively. The 'question-mark' phase has a nature that we cannot establish on the basis of a simple heuristic argument. The logic behind this conjectured phase diagram is the following.

- Suppose that $k^{-2} \ll \rho \ll k^{-1-\alpha}$. Given a plate $(x, o)$, which, without loss of generality, we assume to be in the direction $3_{a}$ (that is, minor axis along direction 3 and major axis along direction 1), there will, typically, be many other plates in the set

$$
J:=x+\left\{\left(y_{1}, y_{2}, y_{3}\right),\left|y_{1}\right|<\frac{k}{2},\left|y_{2}\right|<\frac{k}{2},\left|y_{3}\right|<\frac{k^{\alpha}}{2}\right\}
$$

since the volume of $J$ is of the order $k^{2+\alpha}$ and $\rho k^{2+\alpha} \gg 1$.

By the hard core constraint, the plates whose centers are in $J$ cannot have orientation $1_{a}$ : their orientations can only be $3_{a}, 3_{b}, 1_{b}, 2_{a}$ or $2_{b}$. In general, plates in different directions can coexist within $J$. However, the coexistence is unlikely to happen. In fact, suppose for simplicity that only plates in the directions $3_{a}$ and $3_{b}$ coexist within $J$; then, due to the hard core constraint, one needs to leave a region of volume $\sim k^{2}$, at the interface between the regions occupied by $3_{a}$-plates and $3_{b}$-plates, free of any plate center, an event that is very unlikely, since typically any region of such a volume contains many plate centers (because $\rho k^{2} \gg 1$ ). Similarly, if only plates in the directions $3_{a}$ and $2_{b}$ coexist, one needs to leave a region of volume $\sim k^{1+2 \alpha}$, at the interface between the region occupied by $3_{a}$-plates and $2_{b}$-plates, free of any plate center, an event that is very unlikely, since typically any region of such a volume contains many plate centers (because $\rho k^{1+2 \alpha} \gg 1$ ). Analogously, the coexistence between pairs or triplets of plates in different directions can be shown to be unlikely.

Therefore, a typical plate configuration in $J$ consists of many plates, all in the direction $3_{a}$, with centers distributed approximately uniformly, since their interaction, once we prescribe the direction of their axes, is very weak: they 'just' have a hard core repulsion that prevents a plate from occupying an excluded region of volume $\sim k^{1+\alpha}$ around the center of another plate; on the other hand, any region of volume $k^{1+\alpha}$ within $J$ has very small probability of being occupied at all, because $\rho k^{1+\alpha} \ll 1$.

We can now repeat the same argument for a translate $J^{\prime}$ of $J$ that has intersection of order $k^{2+\alpha}$ with $J$ itself: since the intersection typically contains many plates, all oriented in the direction $3_{a}$, we conclude that also the plates in $J^{\prime}$ are all in the direction $3_{a}$, and their centers are distributed almost uniformly. Proceeding like this, 
we conclude, at least heuristically, that the whole space should be covered mostly by plates in the same direction, namely with both the minor and major axes oriented parallel to each other: such a phase is named biaxial nematic phase, and denoted by the symbol $N_{b}$ in the phase diagram.

- Suppose now that $k^{-1-2 \alpha} \ll \rho \ll k^{-2}$. We proceed as in the previous item: given a plate $(x, o)$, which, without loss of generality, we assume to be in the direction $3_{a}$, there will, typically, be many other plates in the set $J$ defined in (1). By the hard core constraint, as before, the plates whose centers are in $J$ can only be in the directions $3_{a}, 3_{b}, 1_{b}, 2_{a}$ or $2_{b}$. However, in order to accomodate plates with orientation $1_{b}, 2_{a}$ or $2 b$ within $J$, one needs to leave a region of volume $\sim k^{1+2 \alpha}$ free of any plate center, an event that is very unlikely, since typically any region of such a volume contains many plate centers, because $\rho k^{1+2 \alpha} \gg 1$. We conclude that, typically, $J$ contains only plates in the directions $3_{a}$ and $3_{b}$ (that is, of type 3 ): therefore, a typical plate configuration in $J$ consists of many plates, all of type 3 , with centers distributed approximately uniformly, since their interaction, once we prescribe the direction of their minor axes, is very weak: they 'just' have a hard core repulsion that prevents a plate from occupying an excluded region of volume $\sim k^{2}$ around the center of another plate; on the other hand, any region of volume $k^{2}$ within $J$ has very small probability of being occupied at all, because $\rho k^{2} \ll 1$.

In conclusion, we expect that most of the plates in $J$ are oriented in the directions $3_{a}$ or $3_{b}$. Reasoning as in the previous item, we conclude that the whole space should be covered mostly by plates of the same type, that is, with their minor axes aligned, a phase named uni-axial, or plate-like, nematic $\left(N_{-}\right)$phase.

- If $\rho k^{2+\alpha} \ll 1$, then there are few enough plates that they will almost never be in one another's interaction set, so the system is in an isotropic $(I)$ phase.

Note that in the list above there is a 'gap' in the densities: in fact, in the range $k^{-2-\alpha} \ll \rho \ll k^{-1-2 \alpha}$, the reasoning above does not allow us to draw any definite conclusion about the expected nature of the corresponding phase. As far as we know, even numerically, there is no clear evidence about the existence and nature of long range ordering in this range of densities.

Another range of densities that is not discussed in the previous list, is the one very close to close-packing. In analogy with the two-dimensional case [8], we expect no orientational order at very high densities. It would be very interesting to clarify the (glassy?) nature of the very dense phase, and possibly identify a hidden order parameter characterizing its behavior.

We are finally ready to state, informally, our main result. For a more quantitive statement, see the next section.

Main result (informal statement): In the context described above, we consider a system of anisotropic plates of size $1 \times k^{\alpha} \times k$, with $k \gg 1$ and $\frac{3}{4}<\alpha \leq 1$, interacting via purely hard-core interactions. If the density is well within the range where uni-axial nematic ordering is expected, that is, more precisely, if $k^{-3 \alpha} \log k \ll \rho \ll k^{\alpha-3}$ (note that, for $k$ large, and for the values of $\alpha$ under consideration, $k^{-1-2 \alpha} \ll k^{-3 \alpha} \log k$ and $\left.k^{\alpha-3} \leq k^{-2}\right)$, then the system is, in fact, in a uni-axial, plate-like, nematic $\left(N_{-}\right)$phase: in particular, we prove the existence of long range orientational order for the minor axes of the plates, and the absence of translational order, namely, exponential decay of the truncated center-center correlations.

The main idea of the proof is to map the model to a coarse-grained contour model and prove that we can compute its partition function and the expectation of local observables using a convergent cluster expansion. To that end, we will first split the lattice $\Lambda$ into 
cubes of size $\ell:=k / 2$. In the range of densities we are studying, each cube contains, on average, many plates (since $\rho k^{3} \gg 1$ ). We will then define a contour as the union of cubes that either do not contain one and only one type of plates, or that touch other cubes which contain plates of a different type. Our endgame will then be to prove that the presence of contours is unlikely, which will imply the main result.

In essence, contours are unlikely because, as we will show below, the probability that a cube contains plates of different types is low. In order to deduce the unlikeliness of contours from the unlikeliness of the cubes of which it is made, and to control the entropy of the contours, we will use methods coming from the Pirogov-Sinai theory of phase transitions.

The strategy of the proof is very similar to the one of [6], in which a system of hard rods in two dimensions was considered. The main technical novelty lies in the proof that a cube of side $\ell$ containing plates of different types (a 'bad cube') has exponentially small probability in the big parameter $\rho k^{2+\alpha}$. Once this is proved, the rest of the proof follows closely the one in [6] and, therefore, we will not spell out all the details of the proofs, and, instead, refer the reader to [6] in which very similar arguments are expounded.

As far as we know, our result is the first rigorous one for the onset of a nematiclike phase in systems of finite-size particles, with finite-range interactions, in the three dimensional continuum. For previous results, see $[2,4,10,11,20,22]$. We refer to the introduction of [6] for a thorough, comparative, discussion of previous results. See also [12] for a recent proof of the existence of nematic-like order in a monomer-dimer system with attractive interactions.

Our inability to rigorously control the bi-axial nematic phase, as well as the optimal range of densities where uni-axial nematic is expected, is related to the highly anisotropic shape of the excluded regions created by the hard core interaction around any given plate. For instance, consider the range of densities between $k^{-2}$ and $k^{-1-\alpha}$, where bi-axial nematic order is expected. From the heuristic discussion above, it would be tempting to think of the 'uniformly magnetized' regions, where both the axes of the plates are mostly aligned in a common direction, as a union of elementary slabs, each of which is a translate and/or rotation of the region $J$ in (1). Even if natural, this choice creates difficulties in the treatment of the 'transition layers' between different uniformly magnetized regions: these layers, which are the basic constituents of the 'Peierls' contours' generically have a wild geometric shape, which does not allow us to derive simple bounds on their probability, depending only on their volume. At least, the methods of this paper did not allow us to overcome these difficulties: therefore, we limited ourselves to a range of densities where paving the space in cubes allow us to derive effective bounds on the probabilities of the 'transition layers', that is, of the connected components of the union of bad cubes.

\section{The Model and Main Results}

We consider a finite cubic box $\Lambda \subset \mathbb{R}^{3}$ of side $L$, such that $L+2 \ell$ is divisible by $8 \ell$, with $\ell:=k / 2$. This specific choice is technical and is motivated by the definitions of bad regions and contours, see Definitions 2 and 3 below; it is conceptually unimportant, since we will eventually send $L$ to infinity. We recall that plates are anisotropic parallelepipeds of size $1 \times k^{\alpha} \times k$, with $k \gg 1$ and $\alpha \in\left(\frac{3}{4}, 1\right]$, with six possible orientations, as in Fig. 1 . We introduce the following notations. Given a plate $p=(x, o) \in \Lambda \times \mathcal{O}=: \omega_{\Lambda}$, let $R_{p} \subset \mathbb{R}^{3}$ denote the geometric support of the plate. Given $X \subset \mathbb{R}^{3}, p$ is said to belong to $X$ if $x \in X$; $p$ is said to intersect $X(p \cap X \neq \emptyset)$ if $R_{p} \cap X \neq \emptyset$; $p$ is said to be 
contained in $X$ if $R_{p} \subset X$. In addition, given another plate $p^{\prime}, p$ is said to intersect $p^{\prime}$ $\left(p \cap p^{\prime} \neq \emptyset\right)$ if $R_{p} \cap R_{p^{\prime}} \neq \emptyset$.

The grand canonical partition function of the model at activity $z>0$ with open boundary conditions is defined as

$$
Z_{0}(\Lambda)=1+\sum_{n \geq 1} \frac{z^{n}}{n !} \int_{\omega_{\Lambda}} \mathrm{d} p_{1} \cdots \int_{\omega_{\Lambda}} \mathrm{d} p_{n} \varphi\left(p_{1}, \ldots, p_{n}\right)
$$

where $\int_{\omega_{\Lambda}} \mathrm{d} p$ is a shorthand for $\int_{\Lambda} \mathrm{d} x \sum_{o \in \mathcal{O}}$, and

$$
\varphi\left(p_{1}, \ldots p_{n}\right)=\prod_{i<j} \varphi\left(p_{i}, p_{j}\right), \quad \varphi\left(p, p^{\prime}\right)=\left\{\begin{array}{l}
1 \text { if } p \cap p^{\prime}=\emptyset \\
0 \text { if } p \cap p^{\prime} \neq \emptyset
\end{array}\right.
$$

As we shall see below, see the first remark after Theorem 1, fixing the activity is equivalent to fixing the densities, at least in the range of densities we are interested in.

In order to prove the main result, we will pick boundary conditions in such a way that one of the types of plates is favored over the others. We will then construct the various infinite volume states by varying the boundary condition. In order to define the boundary condition, we must introduce some additional notation.

We pave $\Lambda$ by cubes of side $\ell$, called "blocks", and by cubes of side $8 \ell$, called "smoothing cubes" ( the boundary of $\Lambda$ by 1 block). The lattice of the centers of the blocks is a lattice of mesh $\ell$, called $\Lambda^{\prime}$ and the lattice of the centers of the smoothing cubes is a lattice of mesh $8 \ell$, called $\Lambda^{\prime \prime}$. Given $\xi \in \Lambda^{\prime}$, the block centered at $\xi$ is denoted by $\Delta \xi$, and given $a \in \Lambda^{\prime \prime}$, the smoothing cube centered at $a$ is denoted by $\mathcal{S}_{a}$. Given a set $X \subseteq \Lambda$ that is a union of blocks, we denote the coarse-grained version of $X$ by $X^{\prime}$ :

$$
X=\bigcup_{\xi \in X^{\prime}} \Delta_{\xi}
$$

We denote the $L_{\infty}$ distance on $\Lambda$ by

$$
d_{\infty}\left(\left(x_{1}, x_{2}, x_{3}\right),\left(y_{1}, y_{2}, y_{3}\right)\right):=\max \left\{\left|x_{i}-y_{i}\right|, i \in\{1,2,3\}\right\}
$$

and the rescaled $L_{\infty}$ distance on $\Lambda^{\prime}$ by

$$
d_{\infty}^{\prime}(\xi, \eta):=\frac{d_{\infty}(\xi, \eta)}{\ell}
$$

We introduce a coarse-grained spin model on $\Lambda^{\prime}$ : let $\Theta_{\Lambda^{\prime}}$ denote the set of spin configurations $\sigma \equiv\left\{\sigma_{\xi}\right\}_{\xi \in \Lambda^{\prime}}$ with $\sigma_{\xi} \in\{0,1,2,3,4\}$. Given a spin configuration $\sigma \in$ $\Theta_{\Lambda^{\prime}}$ and a plate configuration $P \in \bigcup_{n>0} \omega_{\Lambda}^{n}=: \Omega_{\Lambda}, P$ is said to be compatible with $\sigma$ if it is such that, $\forall \xi \in \Lambda^{\prime}$,

- if $\sigma_{\xi}=0$, then no plates belong to $\Delta_{\xi}$,

- if $\sigma_{\xi}=i$ with $i \in\{1,2,3\}$, then every plate that belongs to $\Delta_{\xi}$ is of type $i$ (this includes the case in which no plates belong to $\Delta_{\xi}$ ),

- if $\sigma_{\xi}=4$, then $\Delta_{\xi}$ contains at least two plates of different type. 
The set of plate configurations that are compatible with a given spin configuration $\sigma$ is denoted by $\Omega_{\Lambda}(\sigma)$. In addition, given a block $\Delta_{\xi}$ and a plate configuration $P$, we denote the restriction of $P$ to $\Delta_{\xi}$ by $P_{\xi}$, and we define the set of $P_{\xi}$ 's that are compatible with $\sigma_{\xi}$ by $\Omega_{\Delta_{\xi}}^{\sigma_{\xi}}$ (for example, $\Omega_{\Delta_{\xi}}^{1} \subset \Omega_{\Delta_{\xi}}$ is the set of plate configurations in $\Delta_{\xi}$ consisting either of plates of type 1 or of the empty configuration). The subset of $\Omega_{\Delta_{\xi}}^{\sigma_{\xi}}$ consisting of configurations with $n$ plates is denoted by $\Omega_{\Delta_{\xi}}^{n, \sigma_{\xi}}$.

We rewrite the grand canonical partition function (2) in $\Lambda$ with open boundary conditions in terms of spin configurations:

$$
Z_{0}(\Lambda)=\sum_{\sigma \in \Theta_{\Lambda^{\prime}}} \int_{\Omega_{\Lambda}(\sigma)} \mathrm{d} P \varphi(P) z^{|P|}
$$

and

$$
\begin{aligned}
& \int_{\Omega_{\Lambda}(\sigma)} \mathrm{d} P:=\prod_{\xi \in \Lambda^{\prime}} \int_{\Omega_{\Delta_{\xi}}^{\sigma_{\xi}}} \mathrm{d} P_{\xi}, \\
& \text { with } \int_{\Omega_{\Delta_{\xi}}^{\sigma_{\xi}}} \mathrm{d} P_{\xi}=\mathfrak{z}_{0}\left(\sigma_{\xi}\right)+\mathbb{1}\left(\sigma_{\xi} \neq 0\right) \sum_{n_{\xi} \geq 1} \frac{1}{n_{\xi} !} \int_{\Omega_{\Delta_{\xi}}^{n_{\xi}, \sigma_{\xi}}} \mathrm{d} p_{1} \cdots d p_{n_{\xi}}
\end{aligned}
$$

in which $\mathbb{1}\left(\sigma_{\xi} \neq 0\right) \in\{0,1\}$ is equal to 1 if and only if $\sigma_{\xi} \neq 0$, and

$$
\mathfrak{z}_{0}(1)=\mathfrak{z}_{0}(2)=\mathfrak{z}_{0}(3)=1, \quad \mathfrak{z}_{0}(0)=-2, \quad \mathfrak{z}_{0}(4)=0 .
$$

The value of $z_{0}$ is the contribution of the empty configuration to the partition function, and the fact that it equals -2 for spin- 0 blocks compensates for the fact that the empty configuration is over-counted by $\sigma_{\xi}=1,2,3$.

We now define the partition function with $q$ boundary conditions, $q \in\{1,2,3\}$, denoted by $Z(\Lambda \mid q)$ :

$$
Z(\Lambda \mid q)=\sum_{\sigma \in \Theta_{\Lambda^{\prime}}^{q}} \int_{\Omega_{\Lambda}(\sigma)} \mathrm{d} P \varphi(P) z^{|P|}
$$

where $\Theta_{\Lambda^{\prime}}^{q} \subset \Theta_{\Lambda^{\prime}}$ is the set of spin configurations, that are such that $\sigma_{\xi}=q$ if $d_{\infty}^{\prime}\left(\xi,\left(\mathbb{Z}^{3} \backslash \Lambda\right)^{\prime}\right) \leq 8$. The number 8 appearing here is related to the choice of smoothing cubes of side $8 \ell$ and to the fact that $L=8 \ell m-2 \ell$, for some integer $m$. This specific choice is motivated by the definitions of good/bad regions and contours, introduced in Sect. 4. In fact, the requirement that $\sigma_{\xi}=q$ if $d_{\infty}^{\prime}\left(\xi,\left(\mathbb{Z}^{3} \backslash \Lambda\right)^{\prime}\right) \leq 8$ is equivalent to the condition that the 'boundary smoothing cubes' (i.e., those intersecting $\Lambda^{c}$ ) are all good with magnetization $q$, that is, all the sampling cubes that they intersect are good and have magnetization $q$, in the sense of Definition 2 .

Remark. The definition of $Z(\Lambda \mid q)$ in (10) does not require that $\Lambda$ is a cube: it holds in the more general case that $\Lambda$ is a connected region obtained by taking a union of smoothing cubes and removing all blocks whose center is at $d_{\infty}^{\prime}$-distance equal to 1 from the complement set.

In the following we will be interested in the $n$-point correlation functions with $q$ boundary conditions, defined as

$$
\rho_{n}^{(q)}\left(p_{1}, \ldots, p_{n}\right):=\lim _{\Lambda \nearrow \mathbb{R}^{3}} \rho_{n}^{(q, \Lambda)}\left(p_{1}, \ldots, p_{n}\right)
$$


with

$$
\rho_{n}^{(q, \Lambda)}\left(p_{1}, \ldots, p_{n}\right):=\frac{1}{Z(\Lambda \mid q)} \sum_{\sigma \in \Theta_{\Lambda^{\prime}}^{q}} \int_{\Omega_{\Lambda}(\sigma)} \mathrm{d} P z^{|P|+n} \varphi\left(\left(p_{1}, \ldots, p_{n}\right) \cup P\right) .
$$

Theorem 1 (Nematic order). Given $\alpha \in\left(\frac{3}{4}, 1\right]$, there exist positive constants $c_{1}, C_{1}, c_{2}$, $c_{3}, c_{4}$, such that if $z k^{3-\alpha} \leq c_{1}$ and $z k^{3 \alpha} / \log k \geq C_{1}$, then for any $q \in\{1,2,3\}$, $\rho_{1}^{(q)}(x, o)$ and $\rho_{2}^{(q)}\left(\left(x_{1}, o_{1}\right),\left(x_{2}, o_{2}\right)\right)$ exist and are invariant under translations, that is, $\rho_{1}^{(q)}(x, o)=\rho_{o}^{(q)}$ and $\rho_{2}^{(q)}\left(\left(x_{1}, o_{1}\right),\left(x_{2}, o_{2}\right)\right)=\rho_{o_{1}, o_{2}}^{(q)}\left(x_{1}-x_{2}\right)$. Moreover, letting

$$
\epsilon:=\max \left\{\left(z k^{2}\right)^{c_{2}}, e^{-c_{3} z k^{2+\alpha}}\right\}
$$

we have, for $m \neq q$,

$$
\rho_{q_{a}}^{(q)}=\rho_{q_{b}}^{(q)}=z(1+O(\epsilon)), \quad \rho_{m_{a}}^{(q)}=\rho_{m_{b}}^{(q)}=O(z \epsilon)
$$

and

$$
\rho_{o_{1}, o_{2}}^{(q)}\left(x_{1}-x_{2}\right)-\rho_{o_{1}}^{(q)} \rho_{O_{2}}^{(q)}=\rho_{o_{1}}^{(q)} \rho_{O_{2}}^{(q)} O\left(\epsilon^{c_{4}\left|x_{1}-x_{2}\right| / k}\right) .
$$

This theorem states that, in the presence of $q$ boundary conditions, most particles are of type $q$ (existence of orientational order), and the truncated two-point correlation function decays exponentially (absence of positional order).

Remark. The proof provides much more detailed information on the set of correlations than what is explicitly stated: in fact, our construction may be applied to the computation of the whole set of correlation functions in terms of a convergent cluster expansion, analogous to the one given in Theorem 2. In particular, the equation for the total density as a function of the activity, of the form $\rho=2 z(1+O(\epsilon))$, can be inverted via the analytic implicit function theorem, and leads to an equation of the form $z=\frac{1}{2} \rho(1+O(\epsilon))$ : therefore, as anticipated above, fixing the activity or the density is equivalent. We also expect that all higher order density correlations satisfy the cluster property, in analogy with the two-point function in (15), hence the infinite volume Gibbs state with $q$ boundary conditions is pure.

Remark. In order to compute the correlation functions, one can replace the activity $z$ with a plate-dependent activity $\tilde{z}(p)$ and express the $n$-point truncated correlation function in terms of the partition function with the modified activities:

$$
\left.z^{n} \frac{\delta^{n}}{\delta \tilde{z}\left(p_{1}\right) \cdots \delta \tilde{z}\left(p_{n}\right)} \log Z(\Lambda \mid q)\right|_{\tilde{z}(p) \equiv z} .
$$

It is, therefore, sufficient to compute the partition function with a plate-dependent activity.

2.1. Strategy of the proof. The proof of our main theorem will be split in several steps, which are summarized here.

1. We first reformulate the model in terms of contours, which interact via an exponentially decaying potential. The contours arise after coarse graining the hard plate system to the spin model introduced above: the contours can be thought of as the transition layers between different uniformly magnetized regions. The interaction between contours is computed using a cluster expansion. 
2. We then map the interacting contour model to a hard core polymer model. In order to compute the pressure of the effective interacting contour model, we perform a Mayer expansion of the multi-contour interaction, to quote D. Brydges [5]: "If at first you do not succeed, then expand and expand again". After this second expansion, the polymer model has a purely hard core interaction.

3. The hard core polymer model can be treated by standard cluster expansion methods, provided the activity of the contours is exponentially small in their size. This is to be expected, because the transition layers contain many bad blocks, i.e., cubes containing more than one plate orientations. The key technical lemma is a proof that the probability of a single bad block is small, under the assumptions of our main theorem (see Lemma 1). Building upon this, we obtain the desired estimate on the activity of the contours. A subtle point is that the contour activities are defined inductively, in the spirit of Pirogov-Sinai theory $[13,18]$ therefore, obtaining the bound on the contour activity from the single-block estimate requires an inductive proof, starting from the smaller contours, and then working our way up to larger ones, which may contain smaller contours in their interior(s).

The proof closely follows that in [6], in which a two-dimensional model of hard rods was considered. The important novelty of the present work is to show that the bad blocks mentioned above are, indeed, unlikely to exist (in [6], the analogous statement was trivial). For this reason, we will first present, in Sect. 3, the proof that bad blocks are improbable, and then present the remaining arguments, omitting those parts that are mere repetitions of [6]. More precisely, in Sect. 4 we introduce the contour and hard core polymer representations for the partition function with constant activities, and in Sect. 5 we prove their convergence. Finally, in Sect. 6, we discuss the minor differences arising in the presence of a plate-dependent activity, which, as remarked above, is required for the computation of correlation functions, and we explain how to prove the bounds (14)-(15).

\section{Bad Blocks and Dipoles}

In this section we prove two basic bounds on the probability of bad blocks (that is, blocks $\Delta_{\xi}$ with spin $\sigma_{\xi}=4$ ) and bad dipoles (that is, pairs of neighboring blocks $\Delta_{\xi}, \Delta_{\eta}$ with spins $\sigma_{\xi}, \sigma_{\eta} \in\{1,2,3\}$ and $\sigma_{\xi} \neq \sigma_{\eta}$ ).

Lemma 1 (Bad blocks). Given a block $\Delta$ which, we recall, is a $k / 2 \times k / 2 \times k / 2$ cube, let $Z^{\geq 2}(\Delta)$ denote the partition function of plate configurations in $\Delta$ containing at least two different types of plates, and, for $q \in\{1,2,3\}$, let $Z^{q}(\Delta)$ denote the partition function of type-q plates in $\Delta$ :

$$
Z^{q}(\Delta)=\int_{\Omega_{\Delta}(q)} \mathrm{d} P \varphi(P) z^{|P|} .
$$

There exist positive constants $c_{5}, c_{6}, C_{2}$ such that, if $z k^{3-\alpha} \leq c_{5}$ and $z k^{3 \alpha} \geq C_{2} \log k$, then

$$
\frac{Z^{\geq 2}(\Delta)}{Z^{q}(\Delta)} \leq e^{-c_{6} z k^{2+\alpha}} .
$$

Proof of Lemma 1. The main idea of the proof is the following. In the uniformly magnetized system, the block $\Delta$ contains many plates in the two directions $q_{a}$ and $q_{b}$. Whenever two types of plates coexist, there must, because of the hard core interaction, be a boundary layer between plates of different types, of thickness $k^{\alpha}$, in which only one of the 
two directions is allowed. The volume of this layer is of the order of $k^{2+\alpha}$, which means that the volume that plates can occupy in $Z^{\geq 2}(\Delta)$ is smaller than that in $Z^{q}(\Delta)$ by $k^{2+\alpha}$. Furthermore, since, as will be shown below, plate partition functions are exponential in the volume of the available space, this yields a gain factor of order $e^{-z k^{2+\alpha}}$.

In order to estimate the partition functions appearing in this proof, a key tool will be the Mayer expansion. The following estimates will often be used. Let $S$ be a subset of $\mathbb{R}^{3}$, not necessarily a union of boxes. Let $\Omega_{S}^{o}$, resp. $\Omega_{S}^{q}$, be the set of plate configurations of orientation $o \in\left\{1_{a}, 1_{b}, 2_{a}, 2_{b}, 3_{a}, 3_{b}\right\}$, resp. of type $q \in\{1,2,3\}$, and center in $S$; we also denote by $\Omega_{S}^{n, o}$, resp. $\Omega_{S}^{n, q}$, the restriction of $\Omega_{S}^{o}$, resp. $\Omega_{S}^{q}$, to the $n$-plate configurations. Then,

$$
\begin{aligned}
& \log \int_{\Omega_{S}^{o}} \mathrm{~d} P \varphi(P) z^{|P|}=|S| z\left(1+O\left(z k^{1+\alpha}\right)\right), \\
& \log \int_{\Omega_{S}^{q}} \mathrm{~d} P \varphi(P) z^{|P|}=2|S| z\left(1+O\left(z k^{2}\right)\right),
\end{aligned}
$$

where $\int_{\Omega_{S}^{o}} \mathrm{~d} P=1+\sum_{n \geq 1} \frac{1}{n !} \int_{\Omega_{S}^{n, o}} \mathrm{~d} p_{1} \cdots d p_{n}$ and $\int_{\Omega_{S}^{q}} \mathrm{~d} P=1+\sum_{n \geq 1} \frac{1}{n !} \int_{\Omega_{S}^{n, q}} \mathrm{~d} p_{1} \cdots$ $d p_{n}$. This result is a simple extension of the convergence theorems proved for identical particle systems in $[9,17,19]$, and follows from the general theory of cluster expansions, discussed at length in many references, among which $[5,7,14,21]$, see also [6, Section 4.2] for a brief introduction. The factors $z k^{1+\alpha}$ and $z k^{2}$ come from the interaction volumes among plates with the same orientation and same type, respectively.

The Mayer expansion allows us to estimate the partition function of uniformly magnetized systems, but may not be used whenever several types of plates coexist. To treat this last case, we proceed as follows. We split the block $\Delta$ into smaller $k^{\alpha} / 2 \times k^{\alpha} / 2 \times k^{\alpha} / 2$ cubes, which we call pebbles. Because of the hard core interaction between plates, each pebble may only contain plates of a single type. Since $z k^{3 \alpha} \gg 1$ each pebble $\delta$ still contains many plates, and the corresponding partition function can be evaluated by a Mayer expansion: for $q=1,2,3$ we have by (19),

$$
Z^{q}(\delta):=\int_{\Omega_{\delta}^{q}} \mathrm{~d} P \varphi(P) z^{|P|}=e^{\frac{1}{4} z k^{3 \alpha}\left(1+O\left(z k^{2}\right)\right)}
$$

where we used the fact that the volume of the pebble is $|\delta|=k^{3 \alpha} / 8$.

Given a configuration of plates in $\Delta$, we color each pebble according to the following.

- Every pebble containing at least one plate of type 1 is colored red, of type 2 is colored green, and of type 3 is colored blue.

- Empty pebbles are colored black.

Every pebble that contains at least two plates with different orientations is called typical. Pebbles that are not typical are called atypical: every such pebble may be empty, or contain only plates with the same orientation. Atypical pebbles owe their name to their low probability: given an atypical pebble $\delta$ and denoting the partition function of atypical configurations in $\delta$ by $Z^{(\mathrm{a})}(\delta):=\sum_{o \in O} \int_{\Omega_{\delta}^{o}} \mathrm{~d} P \varphi(P) z^{|P|}-5$, where 5 compensates the over-counting of empty configurations in the first addend, we have, by (19),

$$
Z^{(\mathrm{a})}(\delta)=6 e^{\frac{1}{8} z k^{3 \alpha}\left(1+O\left(z k^{2}\right)\right)}-5 .
$$


Hence

$$
\frac{Z^{(\mathrm{a})}(\delta)}{Z^{q}(\delta)} \leq 6 e^{-\frac{1}{8} z k^{3 \alpha}\left(1+O\left(z k^{2}\right)\right)} .
$$

The main idea of the proof is to show that, whenever there are at least two types of plates, $\Delta$ must contain a large number of atypical pebbles, from which we will prove (18).

Let us show that, if $\Delta$ contains at least two different types of plates, it contains at least $k^{2(1-\alpha)} / 2$ atypical pebbles: the proof is based on the two following properties of colorings, which follow from simple geometric considerations:

$(* 1)$ given a typical pebble of some color, the three $k / 2 \times k / 2 \times k^{\alpha} / 2$ tiles that are, respectively, orthogonal to directions 1,2 and 3 and contain the pebble, cannot contain a typical pebble of another color.

$(* 2)$ given a non-empty atypical pebble of some color, at least one of the three $k / 2 \times$ $k / 2 \times k^{\alpha} / 2$ tiles that are, respectively, orthogonal to directions 1,2 and 3 and contain the pebble, cannot contain a typical pebble of another color.

We will now separately consider the cases in which there is only one color of typical pebbles, and those in which there are at least two (if there are no typical pebbles then there are, trivially, $k^{3(1-\alpha)}$ atypical pebbles).

We first consider the case in which there is only one color of typical pebbles. By virtue of the fact that $\Delta$ contains at least two types of plates, there exists a non-empty atypical pebble $\delta$ of a different color. By property $(* 2)$, there is at least one $k / 2 \times k / 2 \times k^{\alpha} / 2$ tile containing $\delta$ that only contains atypical pebbles, of which there are $k^{2(1-\alpha)}$.

Next, we consider the case in which there are typical pebbles of at least two different colors. We denote the set of typical red, green and blue pebbles by $R_{t}, G_{t}$ and $B_{t}$, respectively, and their projection in direction 3 onto the lower horizontal face of $\Delta$ (i.e., its 'floor') by $r_{t}, g_{t}$ and $b_{t}$. By property $(* 1), r_{t}, g_{t}$ and $b_{t}$ are disjoint. We assume, without loss of generality, that $R_{t} \neq \varnothing$ and $G_{t} \neq \emptyset$. There exists at least one pebble $\delta_{r}$ in $R_{t}$ above $r_{t}$, and by property $(* 1)$, all the pebbles at the same height as $\delta_{r}$ that are not above $r_{t}$ are atypical: therefore, there are at least $k^{2(1-\alpha)}-\left|r_{t}\right|$ of them. If $\left|r_{t}\right| \leq k^{2(1-\alpha)} / 2$, then we are done. If not, then consider a pebble $\delta_{g}$ in $G_{t}$; by property $(* 1)$, all the pebbles at the same height as $\delta_{g}$ that are above $r_{t}$ are atypical: therefore, there are at least $\left|r_{t}\right|>k^{2(1-\alpha)} / 2$ of them.

Now, given a plate configuration $P$, we split

$$
\Delta=\left(\Delta_{1}^{(\mathrm{t})}(P) \cup \Delta_{2}^{(\mathrm{t})}(P) \cup \Delta_{3}^{(\mathrm{t})}(P)\right) \cup\left(\delta_{1}(P) \cup \cdots \cup \delta_{N}(P)\right)
$$

in which $\Delta_{i}^{(\mathrm{t})}(P)$ is the union of typical pebbles of type $i$, and $\delta_{j}(P)$ is an atypical pebble. By the discussion above, $N \geq k^{2(1-\alpha)} / 2$, whenever $\Delta$ contains at least two types of plates. We thus have

$$
Z^{\geq 2}(\Delta)=\sum_{N=k^{2(1-\alpha)} / 2}^{k^{3(1-\alpha)}} \sum_{\substack{(t) \equiv\left(\Delta_{1}^{(\mathrm{t})}, \Delta_{2}^{(\mathrm{t})}, \Delta_{3}^{(\mathrm{t})}\right) \\ \underline{\delta} \equiv\left(\delta_{1}, \cdots, \delta_{N}\right)}} Z^{*}\left(\underline{\Delta}^{(\mathrm{t})}, \underline{\delta}\right),
$$

in which the sum over $\underline{\Delta}^{(\mathrm{t})}$ and $\underline{\delta}$ is the sum over subsets for which there exists a plate configuration $P$ such that $\underline{\Delta}^{(\mathrm{t})} \equiv \underline{\Delta}^{(\mathrm{t})}(P)$ and $\underline{\delta} \equiv \underline{\delta}(P)$, and $Z^{*}\left(\underline{\Delta}^{(\mathrm{t})}, \underline{\delta}\right)$ is the partition function of plate configurations $P$ such that $\underline{\Delta}^{(\overline{\mathrm{t})}}(P) \equiv \Delta^{(\mathrm{t})}$ and $\underline{\delta}(P) \equiv \underline{\delta}$. Furthermore, 


$$
Z^{*}\left(\underline{\Delta}^{(\mathrm{t})}, \underline{\delta}\right) \leq\left(\prod_{i=1}^{3} Z^{i}\left(\Delta_{i}^{(\mathrm{t})}\right)\right)\left(\prod_{j=1}^{N} Z^{(\mathrm{a})}\left(\delta_{j}\right)\right)
$$

and, by (19) with $|S|=\left|\Delta_{i}^{(\mathrm{t})}\right| \leq k^{3} / 8$, it holds for every $i, q \in\{1,2,3\}$,

$$
Z^{i}\left(\Delta_{i}^{(\mathrm{t})}\right)=Z^{q}\left(\Delta_{i}^{(\mathrm{t})}\right) e^{O\left(z k^{3} z k^{2}\right)} .
$$

We now split the denominator $Z^{q}(\Delta)$, which, we recall, is the partition function with at most one type of plates. By (19),

$$
Z^{q}(\Delta)=\left(\prod_{i=1}^{3}\left(Z^{q}\left(\Delta_{i}^{(\mathrm{t})}\right) e^{O\left(z k^{3} z k^{2}\right)}\right)\right)\left(\prod_{j=1}^{N}\left(Z^{q}\left(\delta_{j}\right) e^{O\left(z k^{3 \alpha} z k^{2}\right)}\right)\right) .
$$

Thus, by (22), (25), (26), (27),

$$
\frac{Z^{*}\left(\Delta^{(t)}, \underline{\delta}\right)}{Z^{q}(\Delta)} \leq e^{O\left(z k^{3} z k^{2}\right)} 6^{N} e^{-\frac{N}{8} z k^{3 \alpha}\left(1+O\left(z k^{2}\right)\right)}
$$

which we plug into (24), thus getting

$$
\frac{Z^{\geq 2}(\Delta)}{Z^{q}(\Delta)} \leq e^{O\left(z k^{3} z k^{2}\right)} \sum_{N=k^{2(1-\alpha)} / 2}^{k^{3(1-\alpha)}}\left(\begin{array}{c}
k^{3(1-\alpha)} \\
N
\end{array}\right) e^{-\frac{N}{8} z k^{3 \alpha}\left(1+O\left(z k^{2}\right)\right)}(6 C)^{N},
$$

for some constant $C>0$. Here $C^{N}\left(\begin{array}{c}k^{3(1-\alpha)} \\ N\end{array}\right)$ is an upper bound for the number of terms in the sum over $\underline{\Delta}^{(t)}$ and $\underline{\delta}$. To see this, an observation that plays a key role is that $\Delta_{1}^{(\mathrm{t})}, \Delta_{2}^{(\mathrm{t})}$ and $\Delta_{3}^{(\mathrm{t})}$ must be mutually disconnected, because of property $(* 1)$ [note: two pebbles that touch at an edge or a corner are considered as disconnected]. Therefore, each connected component of $\Delta^{(t)}=\Delta_{1}^{(\mathrm{t})} \cup \Delta_{2}^{(\mathrm{t})} \cup \Delta_{3}^{(\mathrm{t})}$ is either of type 1 , or 2 , or 3; moreover, it must be adjacent to at least one atypical pebble, which implies that the number of connected components of $\Delta^{(t)}$ is certainly smaller than $6 N$ (here 6 is the number of faces of an atypical pebble). Given these observations, it is easy to count the number of terms in the sum over $\underline{\Delta}^{(t)}$ and $\underline{\delta}$ : in fact, we can first choose the atypical pebbles, which costs a factor smaller or equal than $\left(\begin{array}{c}k^{3(1-\alpha)} \\ N\end{array}\right)$, and then sum over the partitions of $\Delta^{(t)}$ into the three sets $\Delta_{1}^{(\mathrm{t})}, \Delta_{2}^{(\mathrm{t})}, \Delta_{3}^{(\mathrm{t})}$. Such a sum over partitions costs at most a factor $3^{N^{\prime}}$, where $N^{\prime}$ is the number of connected components of $\Delta^{(t)}$, and 3 is the number of 'colors' (that is, 1,2 or 3) that we can attach to each connected component. As observed above, $N^{\prime} \leq 6 N$, so that the constant $C$ in (29) is smaller than $3^{6}$. From (29) we immediately get:

$$
\begin{aligned}
\frac{Z^{\geq 2}(\Delta)}{Z^{q}(\Delta)} \leq & e^{O\left(z k^{3} z k^{2}\right)} e^{-\frac{1}{32} k^{2(1-\alpha)} \cdot z k^{3 \alpha}\left(1+O\left(z k^{2}\right)\right)} \\
& \cdot \sum_{N=0}^{k^{3(1-\alpha)}}\left(\begin{array}{c}
k^{3(1-\alpha)} \\
N
\end{array}\right) e^{-\frac{1}{16} N z k^{3 \alpha}\left(1+O\left(z k^{2}\right)\right)}(6 C)^{N} \\
= & e^{O\left(z k^{3} z k^{2}\right)} e^{-\frac{1}{32} z k^{2+\alpha}\left(1+O\left(z k^{2}\right)\right)}\left(1+6 C e^{-\frac{1}{16} z k^{3 \alpha}\left(1+O\left(z k^{2}\right)\right)}\right)^{k^{3(1-\alpha)}} \\
\leq & \exp \left(-\frac{1}{32} z k^{2+\alpha}\left(1+O\left(z k^{3-\alpha}\right)+O\left(z^{-1} k^{1-4 \alpha} e^{-\frac{1}{17} z k^{3 \alpha}}\right)\right),\right)
\end{aligned}
$$


where the exponent $\frac{1}{17}$ in the last line may be replaced by any exponent smaller than $\frac{1}{16}$, for $z k^{2}$ sufficiently small. The last term can be bounded as follows $z^{-1} k^{1-4 \alpha} e^{-\frac{1}{17} z k^{3 \alpha}}=$ $\frac{1}{z k^{3 \alpha}} k^{1-\alpha} e^{-\frac{1}{17} z k^{3 \alpha}} \ll k^{1-\alpha} e^{-\frac{1}{17} z k^{3 \alpha}}$. This, provided $z k^{3 \alpha} \gg \log k$ and $z k^{3-\alpha} \ll 1$, implies (18).

Corollary 1 (Bad dipoles). Given two blocks $\Delta_{1}$ and $\Delta_{2}$ that have a common face, let $Z^{\geq 2}\left(\Delta_{1} \cup \Delta_{2}\right)$ denote the partition function of plates in $\Delta_{1}$ and $\Delta_{2}$, that are such that $\Delta_{1}$ and $\Delta_{2}$ are uniformly magnetized and have different magnetizations. There exist positive constants $c_{7}, c_{8}, C_{3}$ such that, if $z k^{3-\alpha} \leq c_{7}$ and $z k^{3 \alpha} \geq C_{3} \log k$, then

$$
\frac{Z^{\geq 2}\left(\Delta_{1} \cup \Delta_{2}\right)}{Z^{q}\left(\Delta_{1} \cup \Delta_{2}\right)} \leq e^{-c_{8} z k^{2+\alpha}} .
$$

Proof of corollary 1 . Consider the $k / 2 \times k / 2 \times k / 2$ cube $\Delta$ that has half its volume in $\Delta_{1}$ and half in $\Delta_{2}$. Without loss of generality, we assume that $\Delta_{1}$ is to the left of $\Delta_{2}$ in direction 1 . Since $\Delta_{1}$ and $\Delta_{2}$ are uniformly magnetized and have different magnetizations, the plate configuration restricted to the central cube $\Delta$ either has two plates of different types, or is at least half empty. In the second case, we may assume without loss of generality that all plate centers belong to the left half of the cube. Therefore, we bound

$$
\begin{aligned}
Z^{\geq 2}\left(\Delta_{1} \cup \Delta_{2}\right) & \leq \sum_{\substack{\leq i, j \leq 3: \\
i \neq j}} Z^{i}\left(\Delta_{1} \backslash \Delta\right)\left[2 Z^{1, \emptyset}(\Delta)+Z^{\geq 2}(\Delta)\right] Z^{j}\left(\Delta_{2} \backslash \Delta\right) \\
& =6 Z^{q}\left(\Delta_{1} \backslash \Delta\right)\left[2 Z^{1, \emptyset}(\Delta)+Z^{\geq 2}(\Delta)\right] Z^{q}\left(\Delta_{2} \backslash \Delta\right) e^{O\left(z k^{3} z k^{2}\right)},
\end{aligned}
$$

where $Z^{1, \emptyset}(\Delta)$ is the partition function of plate configurations in $\Delta$, such that the plates are all of the same type, and the right half of the box is empty, i.e., contains no plate centers. Using (19), we find

$$
Z^{1, \emptyset}(\Delta)=3 Z^{q}(\Delta) e^{-\frac{1}{8} z k^{3}+O\left(z k^{3} z k^{2}\right)}
$$

and

$$
Z^{q}\left(\Delta_{1} \cup \Delta_{2}\right)=Z^{q}\left(\Delta_{1} \backslash \Delta\right) Z^{q}(\Delta) Z^{q}\left(\Delta_{2} \backslash \Delta\right) e^{O\left(z k^{3} z k^{2}\right)} .
$$

The corollary then follows directly from these two equations and from Lemma 1.

\section{The Contour Theory}

In this section, we construct first the interacting contour model and then the hard core polymer system that were mentioned above. The basic idea of the construction of the contours is that a spin configuration can be seen as a union of connected 'uniformly magnetized regions' (union of blocks that all have the same spin, equal to $q \in\{1,2,3\}$ ), and boundary regions where either the spin changes, or there are 'defects' (blocks with spin equal to 0 or 4 ). Contours will be defined as structures that comprise information about the location and nature of these boundaries, as well as the value of the spin on either side of the boundary. To make this precise, we must first locate the boundary, which we do by defining the concepts of 'good' and 'bad' regions. 


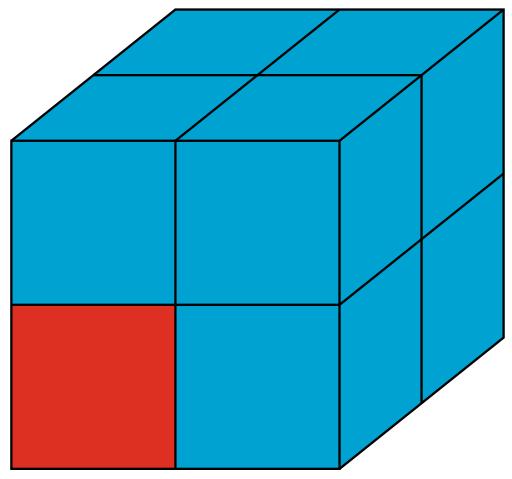

Fig. 3. The sampling cube associated to the red (color online) block

\subsection{Goodness, badness and contours.}

Definition 1 (Sampling cubes). Given $\xi \in \Lambda^{\prime}$, we define the sampling cube associated to $\xi$ as

$$
S_{\xi}=\bigcup_{\substack{\eta \in \Lambda^{\prime} \\ 0 \leq \eta_{i}-\xi_{i} \leq \ell}} \Delta_{\eta}
$$

where $\xi_{i}$ and $\eta_{i}, i=1,2,3$, are the coordinates of $\xi, \eta \in \Lambda^{\prime}$ (see Fig. 3). Note that if $d_{\infty}^{\prime}\left(\xi, \Lambda_{c}^{\prime}\right)>1$, then $S_{\xi}$ contains exactly 8 blocks.

Definition 2 (Good and bad regions). Given a spin configuration $\sigma \in \Theta_{\Lambda^{\prime}}$, a sampling cube $S_{\xi}$ is said to be

$-\operatorname{good}$ if the spins inside $S_{\xi}$ are all equal, and $\sigma_{\xi} \in\{1,2,3\}$. In this case, $\sigma_{\xi}$ is called the magnetization of the sampling cube.

- bad otherwise, that is, every bad sampling cube either contains at least one spin equal to 0 or 4 , or it contains at least one pair of neighboring blocks with different spins.

Furthermore, we define

$$
B(\sigma):=\bigcup_{\xi \in \Lambda^{\prime}: S_{\xi} \text { is bad }} S_{\xi}
$$

as the union of all bad sampling cubes, as well as the coarser set

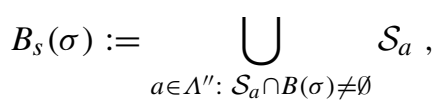

(the lattice $\Lambda^{\prime \prime}$ and the smoothing cubes $\mathcal{S}_{a}$ were defined in Sect. 2). Finally, we define the "bad region" by adding a layer of blocks to $B_{S}$ :

$$
\bar{B}(\sigma)=\bigcup_{\xi \in \Lambda^{\prime}: d_{\infty}^{\prime}\left(\xi, B_{s}(\sigma)\right) \leq 1} \Delta \xi
$$

(see Fig. 4). 


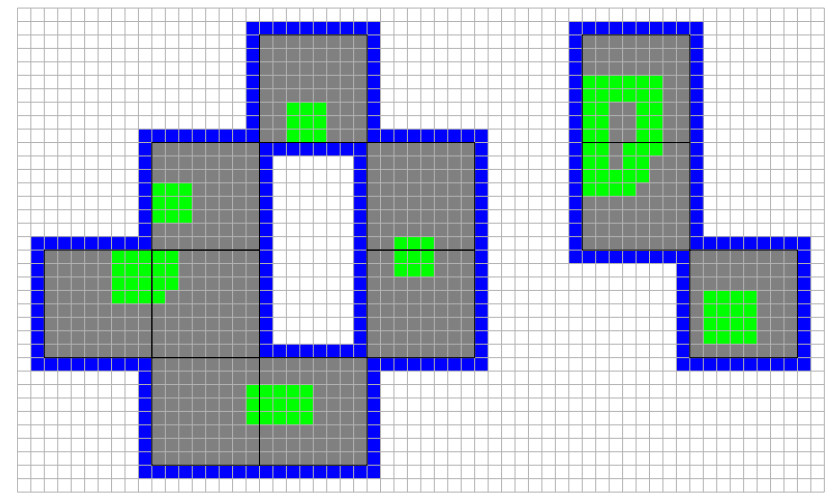

Fig. 4. Example of a bad region (or, rather, a section of a (3-dimensional) bad region): the green (color online) regions are the bad sampling cubes, the gray or green regions are the bad smoothing cubes, and the blue region consists of the extra cubes added in (38)

Remark. In other words, the bad region is a coarse version of the set of blocks which are either empty or contain several plate types, or whose neighbors have a different spin. The reason why we made this set coarser is to ensure that plates in different connected components of $\Lambda \backslash \bar{B}(\sigma)$ do not interact directly, which simplifies the construction of the contour expansion discussed below. Indeed, with our choice, different components are at least at an $L_{\infty}$-distance $2 \ell$, see Fig. 4 . Moreover, our choice guarantees that the distance between two different connected components of $\bar{B}(\sigma)$ is larger than $6 \ell$, which implies that the effective interaction among contours, called $W^{(\Lambda)}(\partial)$ in Lemma 2 below, is conveniently small, since it is mediated by at least three plates; this condition will be used, in particular, in the proof of Lemma 4.

Let $\Gamma$ be one of the connected components of $\bar{B}(\sigma)$. Let $h_{\Gamma}+1 \geq 1$ denote the number of connected components of $\Lambda \backslash \Gamma$. One of these components is adjacent to $\mathbb{Z}^{3} \backslash \Lambda$, and is, naturally, identified as the exterior of $\Gamma$, which we denote by Ext $\Gamma$. When $h_{\Gamma} \geq 1$, the additional connected components of $\Lambda \backslash \Gamma$ are called interiors of $\Gamma$, which we denote by $\operatorname{Int}_{j} \Gamma, j=1, \cdots, h_{\Gamma}$. For future reference, we denote by $\mathfrak{I n t}$ the set of all possible such interiors, as we let the spin configuration $\sigma$ vary in $\Theta_{\Lambda^{\prime}}^{q}$, and the box $\Lambda$ grow. Note that $\Lambda$ is in $\mathfrak{I n t}$, and any element $B \in \mathfrak{I n t}$ satisfies the properties spelled out in the remark after (10). By construction, if $B \in \mathfrak{I n t}$, then $B$ has no interior.

Definition 3 (Contours). Given $q \in\{1,2,3\}$, a spin configuration $\sigma \in \Theta_{\Lambda^{\prime}}^{q}$ and a plate configuration $P \in \Omega_{\Lambda}(\sigma)$, we associate a contour $\gamma:=\left(\Gamma_{\gamma}, \sigma_{\gamma}, P_{\gamma}\right)$ to each connected component of $\bar{B}(\sigma)$. Here

- $\Gamma_{\gamma}$ is the connected component of $\bar{B}(\sigma)$, and is called the support of the contour;

$-\sigma_{\gamma}$ is the restriction of the spin configuration $\sigma$ to $\Gamma_{\gamma}$;

$-P_{\gamma}$ is the restriction of the plate configuration $P$ to $\Gamma_{\gamma}$.

By the definition of $\bar{B}(\sigma)$, all the blocks in

$$
\partial_{\text {ext }} \Gamma_{\gamma}:=\bigcup_{\xi \in \Gamma_{\gamma}^{\prime}: d_{\infty}^{\prime}\left(\xi,\left(\operatorname{Ext} \Gamma_{\gamma}\right)^{\prime}\right)=1} \Delta_{\xi}
$$


have the same magnetization, which we denote by $m_{\mathrm{ext}, \gamma} \in\{1,2,3\}$. Similarly, all the blocks in

$$
\partial_{\text {int }, j} \Gamma_{\gamma}:=\bigcup_{\xi \in \Gamma_{\gamma}^{\prime}: d_{\infty}^{\prime}\left(\xi,\left(\operatorname{Int}_{j} \Gamma_{\gamma}\right)^{\prime}\right)=1} \Delta_{\xi}
$$

have the same magnetization, which we denote by $m_{\mathrm{int}, \gamma}^{j}$. See Fig. 4, where the regions $\partial_{e x t} \Gamma_{\gamma}$ and $\partial_{i n t, j} \Gamma_{\gamma}$ are colored in blue. The collection of all the contours associated with $(\sigma, P)$ is called the contour configuration associated with $(\sigma, P)$ and will be denoted by the symbol $\partial$.

Remark. A contour $\gamma$ must satisfy a number of constraints. For instance:

- $\Gamma_{\gamma}$ must be a union of smoothing cubes, of an external layer and (possibly, if $\left.\cup_{j} \operatorname{Int}_{j} \Gamma_{\gamma} \neq \emptyset\right)$ of internal layers, compatibly with the definition of $\bar{B}(\sigma)$, see (38);

- the spin configuration $\sigma_{\gamma}$ must be such that every sampling cube intersecting $\partial_{\text {ext }} \Gamma_{\gamma}$ is good, with magnetization $m_{\text {ext }, \gamma}$, and similarly for the sampling cubes intersecting $\partial_{\text {int }, j} \Gamma_{\gamma}$;

- the spin configuration $\sigma_{\gamma}$ must be such that each smoothing cube contained in $\Gamma_{\gamma}$ intersects at least one bad sampling cube;

- $P_{\gamma}$ must be compatible with $\sigma_{\gamma}$.

We denote the set of possible contour configurations with $q$ boundary conditions, excluding the empty configuration, by $\mathcal{C}(\Lambda \mid q)$ : this is the set of non-empty contour configurations $\partial$ for which there exist $\sigma \in \Theta_{\Lambda^{\prime}}^{q}$ and $P \in \Omega_{\Lambda}(\sigma)$ such that $\partial$ is the contour configuration associated to $(\sigma, P)$. The contour configurations $\partial \in \mathcal{C}(\Lambda \mid q)$ are fully characterized by the following properties: each $\gamma \in \partial$ is possible (that is, there exist $\sigma \in \Theta_{\Lambda^{\prime}}^{q}$ and $P \in \Omega_{\Lambda}(\sigma)$ such that $\gamma$ is one of the contours associated to $\left.(\sigma, P)\right) ; \Gamma_{\gamma}$ and $\Gamma_{\gamma^{\prime}}$ are disconnected, for all pairs of distinct contours $\gamma, \gamma^{\prime} \in \partial$; the external/internal magnetizations of the contours in $\partial$ satisfy a compatibility condition, namely, if $\Gamma_{\gamma}$, with $\gamma \in \partial$, is immediately contained (We say that $\Gamma_{\gamma}$, with $\gamma \in \partial$, is 'immediately contained' in Int ${ }_{j} \Gamma_{\gamma^{\prime}}$, with $\gamma^{\prime} \in \partial$, if $\Gamma_{\gamma} \subset \operatorname{Int}_{j} \Gamma_{\gamma^{\prime}}$ and there exists no other contour $\gamma^{\prime \prime} \in \partial$ such that $\Gamma_{\gamma} \subset \operatorname{Int}_{j^{\prime}} \Gamma_{\gamma^{\prime \prime}} \subset \operatorname{Int}_{j} \Gamma_{\gamma^{\prime}}$.) in the interior $\operatorname{Int}_{j} \Gamma_{\gamma^{\prime}}$ of another contour $\gamma^{\prime} \in \partial$, then $m_{\text {ext }, \gamma}=m_{\text {int, } \gamma^{\prime}}^{j}$. In terms of these definitions, we can rewrite the partition function (10) as

$$
Z(\Lambda \mid q)=Z^{q}(\Lambda)+\sum_{\partial \in \mathcal{C}(\Lambda \mid q)} \mathbf{Z}_{\partial}(\Lambda \mid q)
$$

where $\mathbf{Z}_{\partial}(\Lambda \mid q)$ denotes the partition function of plate configurations whose associated contour configuration is $\partial$. Note that the sum over $\mathcal{C}(\Lambda \mid q)$ is actually an integral, since it includes an integral over the position of plates inside contour supports. This equality is tautological, and, as such, not all that helpful. Indeed, the compatibility condition among the external/internal magnetizations of the contours, mentioned above, induces an effective long-range interaction between them, which prevents us from using a cluster expansion to compute the partition function of the contour model. This interaction can be eliminated, as stated in Lemma 2 below.

4.2. Interacting contour representation. In addition to $\mathcal{C}(\Lambda \mid q)$, we introduce another set $\mathcal{C}(\Lambda, q)$ of contour configurations. We say that $\partial \in \mathcal{C}(\Lambda, q)$, if the following properties are verified: each contour $\gamma \in \partial$ is possible (in the same sense spelled out above, see a few 
lines above (40)); $\Gamma_{\gamma}$ and $\Gamma_{\gamma^{\prime}}$ are disconnected, for all pairs of distinct contours $\gamma, \gamma^{\prime} \in \partial$; the external magnetization $m_{\text {ext }, \gamma}$ is equal to $q$, for all $\gamma \in \partial$. Note that, by definition, the external magnetization of every contour in a contour configuration $\partial \in \mathcal{C}(\Lambda, q)$ is $q$, even in situations where a contour is immediately contained in another contour whose internal magnetization is different from $q$. Therefore, the contour configurations in $\mathcal{C}(\Lambda, q)$ are not possible contour configurations in the sense given in the previous section, but this is not a problem. On the contrary, summing over the contour configurations in $\mathcal{C}(\Lambda, q)$ is crucial to avoid long-range interactions between contours, thus allowing us to perform a cluster expansion of the contour theory. The desired contour representation of the partition function is summarized in the following lemma.

Lemma 2 (Contour expansion). The conditioned partition function $Z(\Lambda \mid q), q=1,2,3$, can be written as

$$
\frac{Z(\Lambda \mid q)}{Z^{q}(\Lambda)}=1+\sum_{\partial \in \mathcal{C}(\Lambda, q)}\left(\prod_{\gamma \in \partial} \zeta_{q}^{(\Lambda)}(\gamma)\right) e^{W^{(\Lambda)}(\partial)}
$$

where:

$-\zeta_{q}^{(\Lambda)}(\gamma)$ is the activity of $\gamma:$

$$
\zeta_{q}^{(\Lambda)}(\gamma):=\zeta_{q}^{0}(\gamma) \exp \left(-\int_{\Omega_{\Lambda}^{q}} \mathrm{~d} P \varphi^{T}(P) z^{|P|} F_{\gamma}(P)\right)
$$

with

$$
F_{\gamma}(P):=\left\{\begin{array}{l}
1 \text { if there exist two plates } p_{1}, p_{2} \text { in } P \text { such that } \\
\quad p_{1} \text { belongs to } \Lambda \backslash \Gamma_{\gamma} \text { and } p_{2} \text { belongs to } \Gamma_{\gamma}, \\
1 \text { if there exists a plate } p_{1} \text { in } P \text { that belongs to Ext } \Gamma_{\gamma} \\
\text { and a plate } p_{2} \text { in } P_{\gamma} \text { such that } p_{1} \cap p_{2} \neq \emptyset, \\
0 \text { otherwise }
\end{array}\right.
$$

and

$$
\zeta_{q}^{0}(\gamma):=\frac{z^{\left|P_{\gamma}\right|} \varphi\left(P_{\gamma}\right)}{Z^{q}\left(\Gamma_{\gamma}\right)} \prod_{j=1}^{h_{\Gamma}} \frac{Z^{(\gamma)}\left(\operatorname{Int}_{j} \Gamma_{\gamma} \mid m_{\mathrm{int}^{j} \gamma}^{j}\right)}{Z\left(\operatorname{Int}_{j} \Gamma_{\gamma} \mid q\right)},
$$

in which $Z^{(\gamma)}(A \mid m)$, with $A \in \mathfrak{I n t}$ (recall that $\mathfrak{I n t}$ was introduced right before Definition 3), is the partition function of plates in A with $m$-boundary conditions, with the constraint that plates must not intersect plates in $P_{\gamma}$, defined in a way analogous to (10) (cf. also with the remark after (10)):

$$
Z^{(\gamma)}(A \mid m)=\sum_{\sigma \in \Theta_{A^{\prime}}^{m}} \int_{\Omega_{A}(\sigma)} \mathrm{d} P \varphi\left(P \cup P_{\gamma}\right) z^{|P|} .
$$

Moreover, the function $\varphi^{T}(P)$ in (42) is the Ursell function: $\varphi^{T}(\emptyset)=0$ (hence $|P| \geq 1), \varphi^{T}(p)=1$ and, if $n \geq 2$,

$$
\varphi^{T}\left(p_{1}, \cdots, p_{n}\right):=\sum_{\mathfrak{g} \in \mathcal{G}^{T}(n)} \prod_{\left\{j, j^{\prime}\right\} \in \mathcal{E}(\mathfrak{g})}\left(\varphi\left(p_{j}, p_{j^{\prime}}\right)-1\right)
$$


in which $\mathcal{G}^{T}(n)$ is the set of connected graphs on $n$ labeled vertices, and $\mathcal{E}(\mathfrak{g})$ is the set of undirected edges of the graph $\mathfrak{g}$. In particular, $\varphi^{T}(P)$ vanishes if $\bigcup_{p \in P} R_{p}$ is disconnected.

$-W^{(\Lambda)}(\partial)$ is the interaction between the contours in $\partial$ : if $|\partial|=1$, then $W^{(\Lambda)}(\partial)=0$, and if $|\partial| \geq 2$, then

$$
W^{(\Lambda)}(\partial)=\int_{\Omega_{\Lambda}^{q}} \mathrm{~d} P \varphi^{T}(P) z^{|P|} \sum_{n \geq 2} \frac{(-1)^{n}}{n !} \sum_{\gamma_{1}, \cdots, \gamma_{n} \in \partial}^{*} F_{\gamma_{1}}(P) \cdots F_{\gamma_{n}}(P)
$$

where the $*$ on the sum indicates the constraint that $\gamma_{1}, \cdots, \gamma_{n}$ are all distinct.

Remark. Note that $F_{\gamma}(P) \neq 0$ only when either $P$ has two intersecting plates, one of which belongs to the contour's support, and the other to its complement (hence $|P| \geq 2$ ) or $P$ has a plate intersecting one of the plates in the contour (in this case we may have $|P|=1)$.

Remark. Note that the second condition in (43) requires that $p_{1}$ belongs to a block $\Delta_{\xi}$ such that $d_{\infty}^{\prime}\left(\xi, \Gamma_{\gamma}\right) \leq 2$.

Remark. As we will prove in the following, the interaction $e^{W^{(\Lambda)}(\partial)}$ is a short-range interaction, that is, it decays exponentially with the distance between contours. This property is essential to the convergence of the cluster expansion of the contour model.

Remark. For future reference, we note that the constrained partition function in (45) can be rewritten in a form that does not involve summation over spins:

$$
Z^{(\gamma)}(A \mid m)=\int_{\Omega_{\partial A}^{m}} \mathrm{~d} P \varphi\left(P \cup P_{\gamma}\right) z^{|P|} \int_{\Omega_{A^{\circ}}} d \tilde{P} \varphi(\tilde{P} \cup P) z^{|\tilde{P}|},
$$

where $\partial A=\cup_{\xi \in A^{\prime}: d_{\infty}^{\prime}\left(\xi,(\operatorname{Ext} A)^{\prime}\right) \leq 8} \Delta_{\xi}$ is the layer of blocks that are uniformly magnetized by the boundary conditions, and $A^{\circ}=A \backslash \partial A$. On the other hand, this expression is equivalent to

$$
\begin{aligned}
Z^{(\gamma)}(A \mid m) & =\int_{\Omega_{\partial A}^{m} \backslash V_{m}\left(P_{\gamma}\right)} \mathrm{d} P \varphi(P) z^{|P|} \int_{\Omega_{A^{\circ}}} d \tilde{P} \varphi(\tilde{P} \cup P) z^{|\tilde{P}|} \\
& =: Z\left(A \backslash V_{m}\left(P_{\gamma}\right) \mid m\right),
\end{aligned}
$$

where $V_{m}\left(P_{\gamma}\right)$ is the excluded volume created by the plates in $P_{\gamma}$ on those in $P$. Note that $A \backslash V_{m}\left(P_{\gamma}\right)$ is an element of $\mathfrak{I n t}^{\prime}$, where

$$
\mathfrak{I n t}^{\prime}:=\left\{A \backslash V: A \in \mathfrak{I n t} \text { and } V \subset \mathbb{R}^{3} \text { such that } V \subset \bigcup_{\substack{x i \in A^{\prime}: \\ d_{\infty}^{\prime}\left(\xi,(\operatorname{Ext} A)^{\prime}\right) \leq 2}} \Delta \xi .\right.
$$

The idea of the proof is to first map the plate model to one of external contours, which are contour configurations such that a contour may not lie inside another. We then rewrite the partition function as a sum over external contours of the activity of the contour times the partition function inside each contour. Now, the boundary of this partition function is dictated by the internal magnetizations of the contours. To remove this dependence, we replace the boundary condition with $q$, at the price of including an extra factor in the activity of the contour, which is the second ratio in (44). The construction is then 
iterated, and yields a model of contours whose external magnetization is always $q$. This eliminates the long-range interaction between contours. The short-range interaction, mediated by the plates between contours, which are, by construction, all of type $q$, is then computed using a Mayer expansion.

The proof of this lemma is entirely analogous to that of [6, Lemma 1], and is left to the reader.

4.3. Hard core polymer representation. The contours in (41) interact with each other, due to the presence of the many-body potential $W^{(\Lambda)}(\partial)$. In order to set up the cluster expansion, we will first map the interacting contour model to a hard core polymer model. In order to formulate our next technical lemma, we need a couple more definitions. We let $\mathfrak{B}(\Lambda)$ be the set of unions of blocks in $\Lambda$, and $\mathfrak{B}^{T}(\Lambda)$ the set of $D$-connected unions of blocks in $\Lambda$ (with the prefix " $D$ " meaning "diagonal"): here we say that two blocks are $D$-connected if they touch either on a face, or on an edge or at a corner; of course, if they are not $D$-connected, we say that they are $D$-disconnected.

Lemma 3 (Polymer expansion). We have

$$
\frac{Z(\Lambda \mid q)}{Z^{q}(\Lambda)}=1+\sum_{n \geq 1} \frac{1}{n !} \sum_{X_{1}, \ldots, X_{n} \in \mathfrak{B}^{T}(\Lambda)} \phi\left(X_{1}, \ldots, X_{n}\right) \prod_{i=1}^{n} K_{q}^{(\Lambda)}\left(X_{i}\right)
$$

where:

$-\phi\left(\left\{X_{1}, \cdots, X_{m}\right\}\right) \in\{0,1\}$ is equal to 1 if and only if $X_{i}$ and $X_{j}$ are D-disconnected for all $i \neq j$.

$-K_{q}^{(\Lambda)}(X)$ is the activity of $X$ :

$$
K_{q}^{(\Lambda)}(X):=K_{q, 1}^{(\Lambda)}(X)+K_{q, \geq 2}^{(\Lambda)}(X)
$$

with

$$
K_{q, 1}^{(\Lambda)}(X):=\sum_{\substack{\gamma \in \mathcal{C}_{1}(\Lambda, q) \\ \Gamma_{\gamma}=X}} \zeta_{q}^{(\Lambda)}(\gamma)
$$

and

$$
\begin{aligned}
K_{q, \geq 2}^{(\Lambda)}(X):= & \sum_{\substack{X_{0}, X_{1} \in \mathfrak{B}(X) \\
X_{0} \cup X_{1}=X}} \sum_{\substack{\partial \in \mathcal{C}_{\geq 2}(\Lambda, q) \\
\Gamma_{\partial}=X_{0}}}\left(\prod_{\gamma \in \partial} \zeta_{q}^{(\Lambda)}(\gamma)\right) \\
& \cdot \sum_{p \geq 1} \frac{1}{p !} \sum_{\substack{Y_{1}, \cdots, Y_{p} \subset \mathfrak{B}^{T}(X) \\
Y_{1} \cup \cdots \cup Y_{p}=X_{1}}}^{*}\left(\prod_{j=1}^{p}\left(e^{\mathcal{F}_{\partial}\left(Y_{j}\right)}-1\right)\right)
\end{aligned}
$$

in which: $\mathcal{C}_{1}(\Lambda, q)$ and $\mathcal{C}_{\geq 2}(\Lambda, q)$ denote the sets of contour configurations with, respectively, a single contour, and at least two contours; $\Gamma_{\partial} \equiv \bigcup_{\gamma \in \partial} \Gamma_{\gamma}$; the $*$ on the sum indicates that the sets $Y_{1}, \cdots, Y_{p}$ are different from each other;

$$
\mathcal{F}_{\partial}(Y):=\sum_{n \geq 2} \frac{(-1)^{n}}{n !} \sum_{\gamma_{1}, \cdots, \gamma_{n} \subset \partial}^{*} \int_{\Omega_{\Lambda}^{q}} \mathrm{~d} P z^{|P|} \varphi^{T}(P) F_{\gamma_{1}}(P) \cdots F_{\gamma_{n}}(P) \mathfrak{I}_{Y}(P)
$$

where $\mathfrak{I}_{Y}(P) \in\{0,1\}$ is equal to 1 if and only if $Y$ is the smallest D-connected union of blocks that is such that every plate is contained in $Y$ (that is, the support of every plate is a subset of $Y$ ). 
Remark. The sets $Y_{1}, \cdots, Y_{p}$ are not necessarily disconnected, but they are different from each other. By the definitions of $\mathcal{F}_{\partial}(Y)$ and $F_{\gamma}(P)$, it follows that $\mathcal{F}_{\partial}(Y) \neq 0$ (that is, $e^{\mathcal{F}_{\partial}\left(Y_{j}\right)}-1 \neq 0$ ) only if $Y$ is $D$-connected with at least two contours in $\partial$ : in order to prove this fact, it is crucial that every plate is contained in $Y$. Therefore, the sum over $Y_{1}, \ldots, Y_{p}$ in (55) can be restricted to elements in $\mathfrak{B}^{T}(\Lambda)$ that are $D$-connected with at least two contours in $\partial$.

The proof of this lemma is fairly straightforward, and virtually identical to [6, Lemma 2]. The key identity is

$$
\begin{aligned}
e^{W^{(\Lambda)}(\partial)} & =e^{\sum_{n \geq 2} \frac{(-1)^{n}}{n !} \sum_{\gamma_{1}, \cdots, \gamma_{n} \in \partial}^{*} \int_{\Omega_{\Lambda}^{q}} \mathrm{~d} P \varphi^{T}(P) z^{|P|} F_{\gamma_{1}}(P) \cdots F_{\gamma_{n}}(P)} \\
& =\prod_{Y \in \mathfrak{B}^{T}(\Lambda)}\left[\left(e^{\mathcal{F}_{\partial}(Y)}-1\right)+1\right] .
\end{aligned}
$$

The only real difference is that the sets $Y_{i}$ cover all the plates responsible for the interaction between contours, whereas in [6], only the extremal blocks are kept (in [6], the analog of the sets $Y_{i}$ are denoted by $\bar{Y}_{i}$ ). The details are left to the reader.

\section{Convergent Cluster Expansion}

In this section we prove the convergence of the contour expansions introduced above. The results of this section justify a posteriori the definitions given in the previous section, in particular the specific form of the contour representation and of the polymer expansion that we chose and introduced. The key problem is to estimate the contour and polymer activities, which is not trivial, since they involve ratios of partition functions in their interiors, see (44), which must be estimated inductively. Once a smallness condition on the activities is known, the convergence of the expansion is 'trivial', in the sense that it follows from the classical theory of the cluster expansion. The main convergence result of this section is summarized in the following theorem.

Theorem 2 (Polymer cluster expansion). Given $\alpha \in\left(\frac{3}{4}, 1\right]$, if $z k^{3-\alpha}$ and $\log k /\left(z k^{3 \alpha}\right)$ are sufficiently small, then

$$
\left|K_{q}^{(\Lambda)}(X)\right| \leq \bar{\epsilon}^{\left|X^{\prime}\right|}
$$

with

$$
\bar{\epsilon}:=\max \left\{\left(z k^{2}\right)^{c_{9}}, e^{-c_{10} z k^{2+\alpha}}\right\},
$$

for suitable constants $c_{9}, c_{10}>0$. Furthermore,

$$
\log Z(\Lambda \mid q)=\log Z^{q}(\Lambda)+\sum_{n \geq 1} \frac{1}{n !} \sum_{X_{1}, \ldots, X_{n} \in \mathfrak{B}^{T}(\Lambda)} \phi^{T}\left(X_{1}, \ldots, X_{n}\right) \prod_{i=1}^{n} K_{q}^{(\Lambda)}\left(X_{i}\right)
$$

where $\phi^{T}$ is the Ursell function: $\phi^{T}(\emptyset)=0, \phi^{T}(X)=1$ and, if $n \geq 2$,

$$
\phi^{T}\left(X_{1}, \cdots, X_{n}\right):=\sum_{\mathfrak{g} \in \mathcal{G}^{T}(n)} \prod_{\left\{j, j^{\prime}\right\} \in \mathcal{E}(\mathfrak{g})}\left(\phi\left(X_{j}, X_{j^{\prime}}\right)-1\right)
$$

in which $\mathcal{G}^{T}(n)$ is the set of connected graphs on $n$ labeled vertices, and $\mathcal{E}(\mathfrak{g})$ is the set of undirected edges of the graph $\mathfrak{g}$. In particular, $\phi^{T}\left(X_{1}, \ldots, X_{n}\right)$ vanishes if $\bigcup_{i} X_{i}$ is 
D-disconnected. Finally, the sum in the right side of (59) is absolutely convergent: for all $X_{0} \in \mathfrak{B}^{T}(\Lambda), \forall m \geq 1$,

$$
\sum_{n \geq m} \frac{1}{n !} \sum_{X_{1}, \ldots, X_{n} \in \mathfrak{B}^{T}(\Lambda)}\left|\phi^{T}\left(X_{0}, X_{1}, \ldots, X_{n}\right) \prod_{i=1}^{n} K_{q}^{(\Lambda)}\left(X_{i}\right)\right| \leq C\left|X_{0}^{\prime}\right| \bar{\epsilon}^{500 m}
$$

for a suitable constant $C>0$, where $500=10^{3} / 2$ and $(10 \ell)^{3}$ is the value of $|X|$ for the smallest possible contour of non-vanishing activity.

Here we will focus on (57), since the rest of the theorem follows from the general theory of cluster expansions for polymer models, which is standard (see, for instance, $[3,5,7,14,21])$. We will proceed in two steps.

- The first is to prove that, provided the activity $\zeta_{q}^{(\Lambda)}(\gamma)$ of a contour $\gamma$, defined in (42) and (44), decays as $e^{- \text {(const.) } z k^{2+\alpha}\left|\Gamma_{\gamma}^{\prime}\right|}$, then (57) holds. This follows from the fact that the factor $e^{\mathcal{F}_{\partial}\left(Y_{j}\right)}-1$ appearing in (54) is exponentially small in the size of $Y_{j}$, or, in other words, that the interaction between contours is of short range.

- The second step is to prove that $\zeta_{q}^{(\Lambda)}(\gamma)$ is bounded by $e^{- \text {(const.) } z k^{2+\alpha}\left|\Gamma_{\gamma}^{\prime}\right|}$. The basic idea of the proof is that the number of bad blocks or dipoles in $\Gamma_{\gamma}$ is proportional to its rescaled volume $\left|\Gamma_{\gamma}^{\prime}\right|$, and the weight of a bad block or dipole is, by Lemma 1 and Corollary 1 , bounded by $e^{- \text {(const.) } z k^{2+\alpha}}$. A complication comes from the fact that the bound on $\zeta_{q}^{(\Lambda)}(\gamma)$ requires an inductive argument to estimate the ratio of partition functions in (44): for this purpose, we use Theorem 2 inductively, starting from the contours that are so small that their interior cannot contain other contours, and then moving to larger and larger contours.

5.1. Polymer activity. Here we discuss the first step anticipated above: namely, we assume the desired bound on the contour activity, and, on the basis of this hypothesis, we deduce bounds on the polymer activity. From now on, $C, C^{\prime}, \ldots$, and $c, c^{\prime}, \ldots$, indicate universal positive constants (to be thought of as "big" and "small", respectively), whose specific values may change from line to line.

Lemma 4 (Polymer activity). If $z k^{2}$ and $1 /\left(z k^{2+\alpha}\right)$ are sufficiently small and, for every contour $\gamma$,

$$
\left|\int_{\Omega_{\Gamma_{\gamma}}\left(\sigma_{\gamma}\right)} \mathrm{d} P_{\gamma} \zeta_{q}^{(\Lambda)}(\gamma)\right| \leq e^{-c_{11} z k^{2+\alpha}\left|\Gamma_{\gamma}^{\prime}\right|}
$$

for some constant $c_{11}>0$, then the polymer activity satisfies (57), that is,

$$
\left|K_{q}^{(\Lambda)}(X)\right| \leq \bar{\epsilon}^{\left|X^{\prime}\right|}
$$

where $\bar{\epsilon}$ was defined in (58).

Proof of lemma 4. The main idea of the proof is to extract from $\left(e^{\mathcal{F}_{\partial}(Y)}-1\right)$ an exponential decay proportional to $\left(z k^{2}\right)^{c\left|Y^{\prime}\right|}$. This is due to the fact that the only plate configurations $P$ contributing to (55) are the connected ones (here we say that two plates $p, p^{\prime}$ are connected if $\left.p \cap p^{\prime} \neq \emptyset\right)$ : therefore, the number of plates in $P$ must be at least proportional to $\left|Y^{\prime}\right|$. Since, as we will show below, every additional plate after the 
first one in $P$ comes with a factor $z k^{2}$, we find that $\mathcal{F}_{\partial}(Y)$ decays like $\left(z k^{2}\right)^{c\left|Y^{\prime}\right|}$, and similarly for $\left(e^{\mathcal{F}_{\partial}(Y)}-1\right)$. After having extracted this exponential decay, we can insert the bound on $\zeta$ as in (62) and perform the sum over $X_{0}, X_{1}$.

Recalling (52) and (53), we first bound

$$
\left|K_{q, 1}^{(\Lambda)}(X)\right| \leq 5^{\left|X^{\prime}\right|} e^{-c_{11} z k^{2+\alpha}\left|X^{\prime}\right|}
$$

in which the factor 5 comes from enumerating the spin configurations $\sigma_{\gamma}$ in the contour and we used (62) for integrating over the plate configurations at fixed $\sigma_{\gamma}$. This implies the analog of (63) for $K_{q, 1}^{(\Lambda)}(X)$.

The key ingredient in the rest of the proof is the Mayer expansion of the plate model. Once again, we will not discuss this expansion in detail, as it follows from the general theory of cluster expansions $[3,5,7,14,21]$. Recalling the definitions of $\Omega_{S}^{q}$ and $\Omega_{S}^{n, q}$ given right before (19), we let $\Omega_{S}^{\geq l, q}=\cup_{n \geq l} \Omega_{S}^{n, q}$ be the set of plate configurations of type $q$ with center in $S$ and at least $l$ plates. Using a Mayer expansion it can be proved that, for any $S \subset \mathbb{R}^{3}$,

$$
\int_{\Omega_{\Lambda}^{\geq l, q}} \mathrm{~d} P z^{|P|}\left|\varphi^{T}(P)\right| \mathbb{1}\left(p_{1} \text { belongs to } S\right) \leq C^{l} z|S|\left(z k^{2}\right)^{l-1}
$$

for some constant $C>0$, where $p_{1}$ is the first plate in $P$ (recall that the integration measure is symmetric under permutations of the plates in $P$ ). We now want to use this estimate to bound (54). The key point is to estimate the sum over $p$ in the right side of (54). We claim that

$$
\sum_{p \geq 1} \frac{1}{p !} \sum_{\substack{Y_{1}, \cdots, Y_{p} \subset \mathfrak{B}^{T}(X) \\ Y_{1} \cup \cdots \cup Y_{p}=X_{1}}}^{*}\left(\prod_{j=1}^{p}\left|e^{\mathcal{F}_{\partial}\left(Y_{j}\right)}-1\right|\right) \leq\left(z k^{2}\right)^{\frac{c_{0}}{2}\left|X_{1}^{\prime}\right|} e^{\left|X_{0}^{\prime}\right| z k^{3} O\left(z k^{2}\right)} .
$$

To prove this bound we use (65) to estimate $\mathcal{F}_{\partial}(Y)$. Note that the $L_{\infty}$ distance between the centers of two overlapping plates is, at most, $k \equiv 2 \ell$. Since the distance between two disconnected contours is, at least, $6 \ell$, and any plate configuration $P$ contributing to $\mathcal{F}_{\partial}(Y)$ must intersect plates belonging to the supports of at least two disconnected contours (due to the constraints induced by the functions $F_{\gamma_{i}}$ ), then any plate configuration $P$ contributing to $\mathcal{F}_{\partial}(Y)$ must contain at least 3 plates. Moreover, by a similar argument, it must contain at least $1+c_{0}\left|Y^{\prime}\right|$ plates, for a suitable constant $c_{0}$, which can be chosen, e.g., to be $1 / 14$. Note also that $\mathcal{F}_{\partial}(Y)$ is non zero only if $\operatorname{dist}\left(Y, X_{0}\right)=0$, where dist is the Euclidean distance. Therefore, letting: $l_{Y}:=1+\max \left(2, c_{0}\left|Y^{\prime}\right|\right), N$ be the number of contours in $\partial$ that are $D$-connected to the set $Y, \Delta_{\xi_{1}}$ be the 'first' block of $Y$ (with respect to any given order of its blocks) and $S_{Y}$ the union of the sampling cubes intersecting $\Delta \xi_{1}$,

$$
\begin{aligned}
\left|\mathcal{F}_{\partial}(Y)\right| & \leq \sum_{n=2}^{N} \frac{1}{n !} \sum_{\gamma_{1}, \cdots, \gamma_{n} \subset \partial}^{*} \int_{\Omega_{\Lambda}^{\geq l_{Y}, q}} \mathrm{~d} P z^{|P|}\left|\varphi^{T}(P)\right| \mathbb{1}_{p_{1} \text { belongs to } S_{Y}} \mathbb{1}_{\operatorname{dist}\left(Y, X_{0}\right)=0} \\
& \leq 2^{N} z k^{3}\left(C z k^{2}\right)^{\max \left(2, c_{0}\left|Y^{\prime}\right|\right)} \mathbb{1}_{\operatorname{dist}\left(Y, X_{0}\right)=0} \\
& \leq z k^{3}\left(C^{\prime} z k^{2}\right)^{\max \left(2, c_{0}\left|Y^{\prime}\right|\right)} \mathbb{1}_{\operatorname{dist}\left(Y, X_{0}\right)=0}
\end{aligned}
$$


for some constants $C, C^{\prime}>0$, where we used (65) and, in the final bound, we used $N \leq\left|Y^{\prime}\right|$. Moreover we have

$$
\prod_{j=1}^{p}\left|e^{\mathcal{F}_{\partial}\left(Y_{j}\right)}-1\right| \leq e^{\sum_{j=1}^{p}\left|\mathcal{F}_{\partial}\left(Y_{j}\right)\right|} \prod_{j=1}^{p}\left|\mathcal{F}_{\partial}\left(Y_{j}\right)\right|
$$

where

$$
\sum_{j=1}^{p}\left|\mathcal{F}_{\partial}\left(Y_{j}\right)\right| \leq \sum_{\substack{Y \in \mathfrak{B}^{T}\left(X_{1}\right) \\ \operatorname{dist}\left(Y, X_{0}\right)=0}}\left|\mathcal{F}_{\partial}(Y)\right| \leq C^{\prime \prime} z k^{3}\left(z k^{2}\right)^{2}\left|X_{0}^{\prime}\right|
$$

and, using $\sum_{j}\left|Y_{j}^{\prime}\right| \geq\left|X_{1}^{\prime}\right|$,

$$
\prod_{j=1}^{p}\left|\mathcal{F}_{\partial}\left(Y_{j}\right)\right| \leq\left(z k^{2}\right)^{\frac{c_{0}}{2}\left|X_{1}^{\prime}\right|} \prod_{j=1}^{p}\left|\mathcal{F}_{\partial}\left(Y_{j}\right)\right|\left(z k^{2}\right)^{-\frac{1}{2} \max \left(2, c_{0}\left|Y_{j}^{\prime}\right|\right)}
$$

Inserting these estimates in the sum over $p$

$$
\begin{aligned}
& \sum_{p \geq 1} \frac{1}{p !} \sum_{\substack{Y_{1}, \cdots, Y_{p} \subset \mathfrak{B}^{T}(X) \\
Y_{1} \cup \cdots \cup Y_{p}=X_{1}}}^{*} \prod_{j=1}^{p}\left|\mathcal{F}_{\partial}\left(Y_{j}\right)\right|\left(z k^{2}\right)^{-\frac{1}{2} \max \left(2, c_{0}\left|Y_{j}^{\prime}\right|\right)} \\
& \leq \sum_{p \geq 1} \frac{1}{p !}\left(\sum_{\substack{Y \in \mathfrak{B}^{T}(X) \\
\operatorname{dist}\left(Y, X_{0}\right)=0}}\left|\mathcal{F}_{\partial}(Y)\right|\left(z k^{2}\right)^{-\frac{1}{2} \max \left(2, c_{0}\left|Y^{\prime}\right|\right)}\right)^{p} \\
& \leq \exp \left(\sum_{\substack{Y \in \mathfrak{B}^{T}(X) \\
\operatorname{dist}\left(Y, X_{0}\right)=0}}\left|\mathcal{F}_{\partial}(Y)\right|\left(z k^{2}\right)^{-\frac{1}{2} \max \left(2, c_{0}\left|Y^{\prime}\right|\right)}\right) \leq e^{z k^{3}\left|X_{0}^{\prime}\right| O\left(z k^{2}\right)} .
\end{aligned}
$$

Putting the terms together we get (66). Finally, inserting the bound (62) on $\zeta$,

$$
\left|K_{q, \geq 2}^{(\Lambda)}(X)\right| \leq \sum_{\substack{X_{0}, X_{1} \in \mathfrak{B}(X) \\ X_{0} \cup X_{1}=X, X_{0} \neq \emptyset}} 5^{\left|X_{0}^{\prime}\right|} e^{-c_{11} z k^{2+\alpha}\left(1+O\left(z k^{3-\alpha}\right)\right)\left|X_{0}^{\prime}\right|}\left(z k^{2}\right)^{\frac{c_{0}}{2}\left|X_{1}^{\prime}\right|}
$$

where we used $z k^{3} z k^{2}=z k^{2+\alpha} z k^{3-\alpha}$. This yields (63).

5.2. The activity of contours. We will now prove that (62) holds, which proves the convergence of the cluster expansion, and concludes the proof of (57).

Lemma 5 (Contour activity). If $z k^{3-\alpha}$ and $\log k /\left(z k^{3 \alpha}\right)$ are sufficiently small, then

$$
\int_{\Omega_{\Gamma_{\gamma}}\left(\sigma_{\gamma}\right)} \mathrm{d} P\left|\zeta_{q}^{(\Lambda)}(\gamma)\right| \leq e^{-c_{11} z k^{2+\alpha}\left|\Gamma_{\gamma}^{\prime}\right|}
$$

where $c_{11}$ is the same constant appearing in (62). 
Recall (see (42)) that

$$
\begin{aligned}
\int_{\Omega_{\Gamma_{\gamma}}\left(\sigma_{\gamma}\right)} \mathrm{d} P_{\gamma} \zeta_{q}^{(\Lambda)}(\gamma)= & \int_{\Omega_{\Gamma_{\gamma}}\left(\sigma_{\gamma}\right)} \mathrm{d} P_{\gamma}\left(\frac{z^{\left|P_{\gamma}\right|} \varphi\left(P_{\gamma}\right)}{Z^{q}\left(\Gamma_{\gamma}\right)}\right)\left(\prod_{j=1}^{h_{\Gamma_{\gamma}}} \frac{Z^{(\gamma)}\left(\operatorname{Int}_{j} \Gamma_{\gamma} \mid m_{\mathrm{int}, \gamma}^{j}\right)}{Z\left(\operatorname{Int}_{j} \Gamma_{\gamma} \mid q\right)}\right) \\
& \cdot \exp \left(-\int_{\Omega_{\Lambda}^{q}} \mathrm{~d} P \varphi^{T}(P) z^{|P|} F_{\gamma}(P)\right) .
\end{aligned}
$$

In order to prove Lemma 5, we bound each factor in (74), which is done in Lemma 6, 7 and 8 , stated below.

Lemma 6. If $z k^{2}$ is sufficiently small, then

$$
\exp \left(-\int_{\Omega_{\Lambda}^{q}} \mathrm{~d} P \varphi^{T}(P) z^{|P|} F_{\gamma}(P)\right) \leq e^{O\left(z k^{3} z k^{2}\right)\left|\Gamma_{\gamma}^{\prime}\right|}
$$

Lemma 7. If $z k^{3-\alpha}$ and $\log k /\left(z k^{3 \alpha}\right)$ are sufficiently small, then

$$
\int_{\Omega_{\Gamma_{\gamma}}\left(\sigma_{\gamma}\right)} \mathrm{d} P_{\gamma} \frac{z^{\left|P_{\gamma}\right|} \varphi\left(P_{\gamma}\right)}{Z^{q}\left(\Gamma_{\gamma}\right)} \leq e^{-c z k^{2+\alpha}\left|\Gamma_{\gamma}^{\prime}\right|}
$$

for some constant $c>0$.

Lemma 8. If $z k^{3-\alpha}$ and $\log k /\left(z k^{3 \alpha}\right)$ are sufficiently small, then, if $A \in \mathfrak{I n t}$ (recall that Int was introduced right before Definition 3) and $m, q \in\{1,2,3\}$,

$$
\frac{Z(A \mid m)}{Z(A \mid q)} \leq e^{c\left(z k^{3} z k^{2}+\bar{\epsilon}^{C}\right)\left|\left(\partial_{e x t} A\right)^{\prime}\right|}
$$

for some constants $c, C>0$, where $\bar{\epsilon}$ was defined in (58) and $\partial_{\text {ext }} A$ is defined in the same way as (39).

Remark. The constrained partition function $Z^{(\gamma)}\left(\operatorname{Int}_{j} \Gamma_{\gamma} \mid m_{\mathrm{int}, \gamma}^{j}\right)$ appearing in the right side of (74) is smaller than the unconstrained partition function $Z\left(\operatorname{Int}_{j} \Gamma_{\gamma} \mid m_{\text {int, } \gamma}^{j}\right)$. Therefore, Lemma 8 is enough for bounding the ratio $\prod_{j=1}^{h_{\Gamma_{\gamma}}} \frac{Z^{(\gamma)}\left(\operatorname{Int}_{j} \Gamma_{\gamma} \mid m_{\text {int }, \gamma}^{j}\right)}{Z\left(\operatorname{Int}_{j} \Gamma_{\gamma} \mid q\right)}$ in (74). Combining this remark with Lemmas 6, 7 and 8, we obtain Lemma 5.

Proof of Lemma 6. The main idea of the proof is to use the Mayer expansion of the plate model to extract a dominating term, which is negative, and bound the remainder.

We split

$$
-\int_{\Omega_{\Lambda}^{q}} \mathrm{~d} P \varphi^{T}(P) z^{|P|} F_{\gamma}(P)=-\int_{\Omega_{\Lambda}^{1, q}} \mathrm{~d} p z F_{\gamma}(\{p\})-\int_{\Omega_{\Lambda}^{\geq 2, q}} \mathrm{~d} P \varphi^{T}(P) z^{|P|} F_{\gamma}(P)
$$

where we recall that $\Omega_{\Lambda}^{1, q}$ (resp. $\Omega_{\Lambda}^{\geq 2, q}$ ) is the set of plate configurations of type $q$ with 1 plate (resp. at least 2 plates). The first term is non-positive, and the second is bounded by

$$
\left|\int_{\Omega_{\Lambda}^{\geq 2, q}} \mathrm{~d} P \varphi^{T}(P) z^{|P|} F_{\gamma}(P)\right| \leq \int_{\Omega_{\Lambda}^{\geq 2, q}} \mathrm{~d} P\left|\varphi^{T}(P)\right| z^{|P|} \mathbb{1}\left(p_{1} \text { belongs to } S_{\gamma}\right),
$$


where $S_{\gamma}=\cup_{\xi: d_{\infty}^{\prime}\left(\xi, \Gamma_{\gamma}^{\prime}\right) \leq 2} \Delta_{\xi}$ (here we used the second remark after Lemma 2). We have $\left|S_{\gamma}\right| \leq 2\left|\Gamma_{\gamma}\right|$. We are now in the position of applying (65), which gives

$$
\left|\int_{\Omega_{\Lambda}^{\geq 2, q}} \mathrm{~d} P \varphi^{T}(P) z^{|P|} F_{\gamma}(P)\right| \leq O\left(z k^{3} z k^{2}\left|\Gamma_{\gamma}^{\prime}\right|\right) .
$$

Proof of Lemma 7. Let $\sigma_{\gamma}$ be a spin configuration compatible with the fact that $\gamma$ is a contour. As a consequence, every smoothing cube contained in $\Gamma_{\gamma}$ has non zero intersection with at least one bad sampling cube; moreover, by its very definition, each such bad cube must contain either one block with magnetization equal to 0 or 4 , or one pair of neighboring blocks with magnetization $q, q^{\prime} \in\{1,2,3\}$ such that $q \neq q^{\prime}$ (a 'bad dipole' in the sense of Corollary 1 ). Therefore, given $\sigma_{\gamma}$, it is possible to exhibit a partition $\mathcal{P}$ of $\Gamma_{\gamma}$ such that: (i) all the elements of the partition consist either of a single block or of a bad dipole; (ii) if $\mathfrak{M}_{\gamma}$ is the set of blocks in $\mathcal{P}$ with magnetization equal to 0 or 4 and $\mathfrak{D}_{\gamma}$ is the set of bad dipoles in $\mathcal{P}$, then $\left|\mathfrak{M}_{\gamma}\right|+\left|\mathfrak{D}_{\gamma}\right| \geq c^{\prime}\left|\Gamma_{\gamma}^{\prime}\right|$, for a constant $c^{\prime}$ that can be chosen, e.g., equal to $8^{-4}$. We also let $\mathfrak{N}_{\gamma}$ be the set of blocks in $\mathcal{P}$ that are not in $\mathfrak{M}_{\gamma} \cup \mathfrak{D}_{\gamma}$. We then bound

$$
\begin{aligned}
\int_{\Omega_{\Gamma_{\gamma}}\left(\sigma_{\gamma}\right)} \mathrm{d} P_{\gamma} \frac{z^{\left|P_{\gamma}\right|} \varphi\left(P_{\gamma}\right)}{Z^{q}\left(\Gamma_{\gamma}\right)} \leq & \left(\prod_{\mu \in \mathfrak{M}_{\gamma}} \frac{2+Z_{\geq 2}(\mu)}{Z^{q}(\mu)}\right)\left(\prod_{\delta \in \mathfrak{D}_{\gamma}} \frac{Z_{\geq 2}(\delta)}{Z^{q}(\delta)}\right) \\
& \left(\prod_{n \in \mathfrak{N}_{\gamma}} \frac{Z^{\sigma_{n}}(n)}{Z^{q}(n)}\right) e^{O\left(z k^{3} z k^{2}\right)\left|\Gamma_{\gamma}^{\prime}\right|}
\end{aligned}
$$

where the 2 in the first factor in the right side is due to the activity associated with spin 0 , see (9), and the factor $e^{O\left(z k^{3} z k^{2}\right)\left|\Gamma_{\gamma}^{\prime}\right|}$ comes from splitting $Z^{q}$ into blocks and dipoles, as per (19). We now use Lemma 1 and Corollary 1 , and note that $Z^{\sigma_{n}}(n)=Z^{q}(n)$, thus getting

$$
\int_{\Omega_{\Gamma_{\gamma}}\left(\sigma_{\gamma}\right)} \mathrm{d} P_{\gamma} \frac{z^{\left|P_{\gamma}\right|} \varphi\left(P_{\gamma}\right)}{Z^{q}\left(\Gamma_{\gamma}\right)} \leq e^{-c^{\prime \prime} z k^{2+\alpha}\left(\left|\mathfrak{M}_{\gamma}\right|+\left|\mathfrak{D}_{\gamma}\right|\right)} e^{O\left(z k^{2+\alpha} z k^{3-\alpha}\right)\left|\Gamma_{\gamma}^{\prime}\right|}
$$

for some constant $c^{\prime \prime}>0$. The result follows from $\left|\mathfrak{M}_{\gamma}\right|+\left|\mathfrak{D}_{\gamma}\right| \geq c^{\prime}\left|\Gamma_{\gamma}^{\prime}\right|$.

Sketch of the proof of Lemma 8. The main idea of the proof is the following. If $A$ did not contain any contours, it would only contain a single type of plates, and we would be able to express its partition function using a convergent Mayer expansion, and find that the ratio of partition functions only involves clusters that straddle the boundary of $A$. This gives us the appropriate bound, since clusters with at least two plates contribute a weight $z k^{3} z k^{2}$. When $A$ contains contours, we proceed by induction and use the fact that, by the inductive hypothesis, the polymer theory inside $A$ admits a convergent cluster expansion. We then show that the only polymer clusters that contribute to the ratio of partition functions are those that straddle the boundary.

The details of the proof are in direct analogy with the proof of [6, Lemma 5], and are left to the reader. 


\section{Nematic Order}

In this section, we give the proof of Theorem 1, which follows from a simple modification of the cluster expansion in Theorem 2 . We recall that in order to compute density correlations, we need to promote the activity to be plate-dependent, that is, it is a function $\tilde{z}(p)$. The expansions described in the previous sections hold also in this case with the natural modifications, mostly of notational nature.

We first prove the estimate on the 1-point function, (14). Let $p_{0}=\left(x, m_{i}\right)$, with $x \in \mathbb{R}^{3}$ and $m_{i} \in\left\{1_{a}, 1_{b}, 2_{a}, 2_{b}, 3_{a}, 3_{b}\right\}$. Recall the definition of the 1-point correlation function $\rho_{1}^{(q, \Lambda)}\left(p_{0}\right)$ in the state with $q$ boundary conditions, given in (12). Using (16), we can write it as

$$
\begin{aligned}
\rho_{1}^{(q, \Lambda)}\left(p_{0}\right) & =\left.z \frac{\delta}{\delta \tilde{z}\left(p_{0}\right)} \log Z(\Lambda \mid q)\right|_{\tilde{z}(p) \equiv z} \\
& =\left.z \frac{\delta}{\delta \tilde{z}\left(p_{0}\right)} \log Z^{q}(\Lambda)\right|_{\tilde{z}(p) \equiv z}+\left.z \frac{\delta}{\delta \tilde{z}\left(p_{0}\right)} \log \frac{Z(\Lambda \mid q)}{Z^{q}(\Lambda)}\right|_{\tilde{z}(p) \equiv z}
\end{aligned}
$$

The Mayer expansion of the plate model implies that

$$
\left.z \frac{\delta}{\delta \tilde{z}\left(p_{0}\right)} \log Z^{q}(A)\right|_{\tilde{z}(p) \equiv z}=\delta_{m, q} z\left(1+O\left(z k^{2}\right)\right)
$$

for all finite $A \subset \mathbb{R}^{3}$, uniformly in $A$, hence in particular for $A=\Lambda$. The analogue of (14) at finite volume follows from the following lemma. Eq.(14) then follows from taking the limit $\Lambda \nearrow \mathbb{R}^{3}$, which is easily obtained, using the uniform convergence of the Mayer and polymer expansions.

Lemma 9. Let $p_{0}$ be as above, $q \in\{1,2,3\}$ and $A \in \mathfrak{I n t}^{\prime}$, see (50). If the constant $\bar{\epsilon}$ in (58) is sufficiently small, then

$$
\left|z \frac{\delta}{\delta \tilde{z}\left(p_{0}\right)} \log \frac{Z(A \mid q)}{Z^{q}(A)}\right|_{\tilde{z}(p) \equiv z} \mid \leq z O\left(\bar{\epsilon}^{C}\right) \mathbb{1}(A \ni x),
$$

for some $C>0$, uniformly in A.

Proof. We argue by induction on the size of $A$ or, more precisely, in the volume of $\bar{A}$, which is the smallest set in $\mathfrak{I n t}$ containing $A$. If $\bar{A}$ is so small that $A$ cannot contain any contours, then $Z(A \mid q)=Z^{q}(A)$ and (85) is trivially true. Assume now by induction that (85) holds for all $a \in \mathfrak{I n t}^{\prime}$ such that $|\bar{a}|<|\bar{A}|$, and let us prove (85). By the analogue of Theorem 2 with $\Lambda$ replaced by $A \in \mathfrak{I n t}^{\prime}$ and plate-dependent activities,

$$
\begin{aligned}
\left.z \frac{\delta}{\delta \tilde{z}\left(p_{0}\right)} \log \frac{Z(A \mid q)}{Z^{q}(A)}\right|_{\tilde{z}(p) \equiv z}= & \sum_{n \geq 0} \frac{1}{n !} \sum_{X_{0}, \ldots, X_{n} \in \mathfrak{B}^{T}(\bar{A})} \phi^{T}\left(X_{0}, \ldots, X_{n}\right) \\
& \left.z \frac{\delta}{\delta \tilde{z}\left(p_{0}\right)} K_{q}^{(A)}\left(X_{0}\right)\right|_{\tilde{z}(p) \equiv z_{i=1}} \prod_{q}^{n} K_{(A)}^{(A)}\left(X_{i}\right) .
\end{aligned}
$$

We claim that $\left.\frac{\delta}{\delta \tilde{z}\left(p_{0}\right)} K_{q}^{(A)}(X)\right|_{\tilde{z}(p) \equiv z}$ admits a bound similar to the one for $K_{q}^{(A)}(X)$, namely

$$
\left|\frac{\delta}{\delta \tilde{z}\left(p_{0}\right)} K_{q}^{(A)}(X)\right|_{\tilde{z}(p) \equiv z} \mid \leq \bar{\epsilon}^{c\left|X^{\prime}\right|} e^{-m \operatorname{dist}^{\prime}\left(X^{\prime}, \xi_{x}\right)},
$$


for some constants $c, m>0$, where $\xi_{x}$ is the center of the block containing $x$. Inserting (87) in (86), together with $\left|K_{q}^{(A)}(X)\right| \leq \bar{\epsilon}^{\left|X^{\prime}\right|}$, the result follows. We are left with proving (87).

Recall that $K_{q}^{(A)}(X)=K_{q, 1}^{(A)}(X)+K_{q, \geq 2}^{(A)}(X)$. We consider $K_{q, 1}^{(A)}(X)$ first. Using the definition (53), we need to estimate

$$
\frac{\delta}{\delta \tilde{z}\left(p_{0}\right)} K_{q, 1}^{(A)}(X):=\sum_{\substack{\gamma \in \mathcal{C}_{1}(\Lambda, q) \\ \Gamma_{\gamma}=X}} \frac{\delta}{\delta \tilde{z}\left(p_{0}\right)} \zeta_{q}^{(A)}(\gamma)
$$

for $\tilde{z}\left(p_{0}\right)=z$. Recall that

$$
\zeta_{q}^{(A)}(\gamma)=\left(\frac{z^{\left|P_{\gamma}\right|} \varphi\left(P_{\gamma}\right)}{Z^{q}\left(\Gamma_{\gamma}\right)}\right)\left(e^{-\int_{\Omega_{A}^{q}} \mathrm{~d} P \varphi^{T}(P) z^{|P|} F_{\gamma}(P)}\right)\left(\prod_{j=1}^{h_{\Gamma}} \frac{Z^{(\gamma)}\left(\operatorname{Int}_{j} \Gamma_{\gamma} \mid m_{\mathrm{int}, \gamma}^{j}\right)}{Z\left(\operatorname{Int}_{j} \Gamma_{\gamma} \mid q\right)}\right) .
$$

If the derivative hits the first parenthesis, then it can either act on $z^{\left|P_{\gamma}\right|}$, in which case it produces an indicator function $\mathbb{1}\left(P_{\gamma} \ni p_{0}\right)$, or on $Z^{q}\left(\Gamma_{\gamma}\right)$, in which case it generates an extra factor $\frac{\delta}{\delta \tilde{z}\left(p_{0}\right)} \log Z^{q}\left(\Gamma_{\gamma}\right)$, which by Mayer expansion is equal to $\mathbb{1}\left(x \in \Gamma_{\gamma}\right) \delta_{m q}(1+$ $\left.O\left(z k^{2}\right)\right)$.

If the derivative hits the second parenthesis, then it produces an extra factor

$$
-\frac{\delta}{\delta \tilde{z}\left(p_{0}\right)} \int_{\Omega_{A}^{q}} \mathrm{~d} P \varphi^{T}(P) z^{|P|} F_{\gamma}(P)
$$

whose absolute value is bounded from above by $C\left(z k^{2}\right)^{\max \left\{0, c \operatorname{dist}^{\prime}\left(\xi_{x}, \Gamma_{\gamma}^{\prime}\right)-1\right\}} \delta_{m q}$, for some $C, c>0$.

If the derivative hits the third parenthesis, we get extra factors of the form

$$
\frac{\delta}{\delta \tilde{z}\left(p_{0}\right)} \log \frac{Z\left(A_{j}^{\gamma} \mid m^{j}\right)}{Z^{m^{j}}\left(A_{j}^{\gamma}\right)}-\frac{\delta}{\delta \tilde{z}\left(p_{0}\right)} \log \frac{Z\left(A_{j} \mid q\right)}{Z^{q}\left(A_{j}\right)}+\frac{\delta}{\delta \tilde{z}\left(p_{0}\right)} \log \frac{Z^{m^{j}}\left(A_{j}^{\gamma}\right)}{Z^{q}\left(A_{j}\right)}
$$

where, for short, we denoted $A_{j}=\operatorname{Int}_{j} \Gamma_{\gamma}, m^{j}=m_{\text {int }, \gamma}^{j}, A_{j}^{\gamma}=\operatorname{Int}_{j} \Gamma_{\gamma} \backslash V_{m^{j}}\left(P_{\gamma}\right)$ and $V_{m^{j}}\left(P_{\gamma}\right)$ is the excluded volume produced by the plates in $P_{\gamma}$. Now, the first two terms are estimated by the inductive assumption. The third term can be computed explicitly via Mayer expansion, and equals $\delta_{m, m^{j}}\left(1+O\left(z k^{2}\right)\right) \mathbb{1}\left(x \in A_{j}^{\gamma}\right)-\delta_{m, q}\left(1+O\left(z k^{2}\right)\right) \mathbb{1}(x \in$ $\left.A_{j}\right)$.

Putting things together we get

$$
\left|\frac{\delta}{\delta \tilde{z}\left(p_{0}\right)} K_{q, 1}^{(A)}(X)\right| \leq C \bar{\epsilon}^{\left|X^{\prime}\right|} e^{-m \operatorname{dist}^{\prime}\left(\left(X \cup_{j} \operatorname{Int}_{j} X\right)^{\prime}, \xi_{x}\right)},
$$

for some $C, m>0$. Now, note that, if $x \in \cup_{j} \operatorname{Int}_{j} X$, in which case $\operatorname{dist}^{\prime}\left(\left(X \cup_{j}\right.\right.$ $\left.\left.\operatorname{Int}_{j} X\right)^{\prime}, \xi_{x}\right)=0$, then $\left|X^{\prime}\right| \geq c \operatorname{dist}^{\prime}\left(X^{\prime}, \xi_{x}\right)$ for some $c>0$, hence (87) holds for $K_{q, 1}^{(A)}(X)$. 
Finally, we consider $K_{q, \geq 2}^{(A)}(X)$. Using the definition (54) we see that the derivative generates factors of the same form as above, plus an additional factor arising from the derivative of $\mathcal{F}_{\partial}\left(Y_{j}\right)$. By repeating the strategy leading to (67), we get

$$
\left|\frac{\delta}{\delta \tilde{z}\left(p_{0}\right)} \mathcal{F}_{\partial}\left(Y_{j}\right)\right| \leq \delta_{m q} \mathbb{1}\left(x \in Y_{j}\right)\left(C^{\prime} z k^{2}\right)^{\max \left(2, c_{0}\left|Y^{\prime}\right|\right)} \mathbb{1}_{\operatorname{dist}\left(Y, X_{0}\right)=0} .
$$

This leads to the desired bound, (87), for $K_{q, \geq 2}^{(A)}(X)$ and concludes the proof of the lemma.

The computation of the 2-point correlation function is quite similar: let $p_{1}=\left(x_{1}, o_{1}\right)$ $p_{2}=\left(x_{2}, o_{2}\right)$. We write

$$
\rho_{2}^{(q, \Lambda)}\left(p_{1}, p_{2}\right)-\rho_{1}^{(q, \Lambda)}\left(p_{1}\right) \rho_{1}^{(q, \Lambda)}\left(p_{2}\right)=\left.z^{2} \frac{\delta^{2}}{\delta \tilde{z}\left(p_{1}\right) \delta \tilde{z}\left(p_{2}\right)} \log Z(\Lambda \mid q)\right|_{\tilde{z}(p) \equiv z}
$$

and $\log Z(\Lambda \mid q)=\log Z^{q}(\Lambda)+\log \frac{Z(\Lambda \mid q)}{Z^{q}(\Lambda)}$, so that the derivative produces two terms: the first is the second derivative of $\log Z^{q}(\Lambda)$, which we compute using the Mayer expansion, and the second is similar to the right side of (86), with two derivatives rather than one. These two derivatives have the effect of pinning the clusters of polymers to both $x_{1}$ and $x_{2}$ and, because of the exponential decay of their activity, this implies the exponential decay in (15). The details are left to the reader. This concludes the proof of Theorem 1.

Acknowledgements. A.G. and M.D. acknowledge support from the European Research Council (ERC) under the European Union's Horizon 2020 research and innovation programme (ERC CoG UniCoSM, grant agreement n.724939). The work of I.J. was supported by The Giorgio and Elena Petronio Fellowship Fund and The Giorgio and Elena Petronio Fellowship Fund II. The work of M.D. was also supported by the German Research Foundation through the Collaborative Research Center 1060 "The Mathematics of Emergent Effects", project A08.

Open Access This article is distributed under the terms of the Creative Commons Attribution 4.0 International License (http://creativecommons.org/licenses/by/4.0/), which permits unrestricted use, distribution, and reproduction in any medium, provided you give appropriate credit to the original author(s) and the source, provide a link to the Creative Commons license, and indicate if changes were made.

Publisher's Note Springer Nature remains neutral with regard to jurisdictional claims in published maps and institutional affiliations.

\section{References}

1. Acharya, B.R., Primak, A., Kumar, S.: Biaxial nematic phase in bent-core thermotropic mesogens. Phys. Rev. Lett. 92, 14 (2004)

2. Angelescu, N., Zagrebnov, V.A.: A lattice model of liquid crystals with matrix order parameter. J. Phys. A Math. Gen. 15(11), L639-L643 (1982)

3. Bovier, A., Zahradník, M.: J. Stat. Phys. 100(3/4), 765-778 (2000)

4. Bricmont, J., Kuroda, K., Lebowitz, J.L.: The structure of gibbs states and phase coexistence for nonsymmetric continuum widom rowlinson models. Zeitschrift für Wahrscheinlichkeitstheorie und Verwandte Gebiete 67(2), 121-138 (1984)

5. Brydges, D.C.: A short course on cluster expansions (1986)

6. Disertori, M., Giuliani, A.: The nematic phase of a system of long hard rods. Commun. Math. Phys. 323(1), 143-175 (2013)

7. Gallavotti, G., Bonetto, F., Gentile, G.: Aspects of the Ergodic, Qualitative and Statistical Properties of Motion. Springer, Berlin (2004) 
8. Ghosh, A., Dhar, D.: On the orientational ordering of long rods on a lattice. Europhys. Lett. 78(2), 20003 (2007)

9. Groeneveld, J.: Two theorems on classical many-particle systems. Phys. Lett. 3(1), 50-51 (1962)

10. Heilmann, O.J., Lieb, E.H.: Lattice models for liquid crystals. J. Stat. Phys. 20(6), 679-693 (1979)

11. Ioffe, D., Velenik, Y., Zahradník, M.: Entropy-driven phase transition in a polydisperse hard-rods lattice system. J. Stat. Phys. 122(4), 761-786 (2006)

12. Jauslin, I., Lieb, E.H.: Nematic liquid crystal phase in a system of interacting dimers and monomers. Commun. Math. Phys. 363(3), 955-1002 (2018)

13. Kotecký, R., Preiss, D.: An inductive approach to the pirogov-sinai theory. Proceedings of the 11th Winter School on Abstract Analysis, Rendiconti del Circolo Metematico di Palermo, Serie II, supplemento, vol. 3, pp. 161-164 (1984)

14. Kotecký, R., Preiss, D.: Cluster expansion for abstract polymer models. Commun. Math. Phys. 103(3), 491-498 (1986)

15. Madsen, L.A., Dingemans, T.J., Nakata, M., Samulski, E.T.: Thermotropic biaxial nematic liquid crystals. Phys. Rev. Lett. 92, 14 (2004)

16. Merkel, K., Kocot, A., Vij, J.K., Korlacki, R., Mehl, G.H., Meyer, T.: Thermotropic biaxial nematic phase in liquid crystalline organo-siloxane tetrapodes. Phys. Rev. Lett. 93, 23 (2004)

17. Penrose, O.: Convergence of fugacity expansions for fluids and lattice gases. J. Math. Phys. 4(10), 1312$1320(1963)$

18. Pirogov, S.A., Sinai, Y.G.: Phase diagrams of classical lattice systems. Theor. Math. Phys. 25(3), 1185$1192(1975)$

19. Ruelle, D.: Correlation functions of classical gases. Ann. Phys. 25(1), 109-120 (1963)

20. Ruelle, D.: Existence of a phase transition in a continuous classical system. Phys. Rev. Lett. 27(16), 1040-1041 (1971)

21. Ruelle, D.: Statistical Mechanics: Rigorous Results. World Scientific, Singapore (1999)

22. Zagrebnov, V.: Long-range order in a lattice-gas model of nematic liquid crystals. Physica A Stat. Mech. Appl. 232(3-4), 737-746 (1996)

Communicated by H. Spohn 